\title{
Generalized Space Time Block Coded Spatial Modulation for Open-Loop Massive MIMO Downlink Communication Systems
}

\author{
Lixia Xiao, Member, IEEE, Da Chen, Member, IEEE, Ibrahim Hemadeh, Member, IEEE, Pei Xiao, Senior \\ Member, IEEE, and Tao Jiang, Fellow, IEEE
}

\begin{abstract}
In this paper, we propose a generalized space-time block coded spatial modulation (GSTBC-SM) scheme for openloop massive multiple-input and multiple-output (MIMO) downlink communication systems. Specifically, we firstly partition the information bits into multiple groups with each group modulated by the spatial modulation (SM), where the SM symbols are invoked for orthogonal STBC (OSTBC) and quasi-orthogonal STBC (Q-OSTBC) structures. Then, message passing (MP) and block minimum mean square equalization (B-MMSE) detectors are designed for our GSTBC-SM systems, to achieve near-optimal performance with significantly reduced complexity in massive MIMO configurations. Finally, we derive the theoretical average bit error probability (ABEP) of the proposed scheme. The main contribution is that the propose scheme achieves high transmission rate and diversity gain even with small number of radio frequency $(\mathbf{R F})$ chains at the transmitter. Simulation results verify the theoretical derivations and show that the proposed GSTBCSM scheme provides near $20 \mathrm{~dB}$ gain over the conventional GSTBC scheme under massive MIMO configurations.

Index Terms-Spatial Modulation (SM), Space Time Block Coding (STBC), High throughput, Diversity gain.
\end{abstract}

\section{INTRODUCTION}

$\mathbf{S}$ PACE time block coding (STBC), which transmits identical data information via different space and time domains to attain a high diversity gain, has been considered as the key multiple input multiple output (MIMO) technique in wireless communications [1]. There are two classic categories of conventional STBC schemes, i.e., orthogonal STBC (OSTBC) [2]-[7] and quasi-orthogonal STBC (Q-OSTBC) schemes [8][14]. Specifically, Alamouti first proposed the OSTBC scheme with $N_{t}=2$ transmit antennas (TAs) in [2], which has been widely used in mulicarrier systems [3][4]. Then, the authors of [5]-[7] further extended the OSTBC concept into other numbers of TAs. Meanwhile, the Q-OSTBC schemes with a small number of TAs were developed in [8]-[14]. With the

This work was supported in part by the National Key R\&D Program of China with Grant number 2019YFB180003400, National Science Foundation of China with Grant number 61831013, 61631015, and Innovation Fund of WNLO.

L. Xiao is with the Wuhan National Laboratory for Optoelectronics, Huazhong University of Science and Technology, Wuhan 430074, China.(email: lixiaxiao@hust.edu.cn)

D. Chen, and T. Jiang are with Wuhan National Laboratory for Optoelectronics, School of Electronic Information and Communications, Huazhong University of Science and Technology, Wuhan 430074, China (e-mail: chenda@ hust.edu.cn; Tao.Jiang@ieee.org).

I. Hemadeh and P. Xiao are with 5G Innovation Center of University of Surrey, UK (e-mail: ibrahimhemadeh@gmail.com; p.xiao@surrey.ac.uk). requirements of high throughput and reliability for wireless communications, massive MIMO has been considered as the key technique in the next generation communication [15][17]. However, conventional STBC schemes are unsuitable for massive MIMO systems since they significantly complicate the code design and increase the cost of radio frequency (RF) chains under massive MIMO configurations. As a result, it is urgent to design new STBC schemes for massive MIMO systems to improve the throughput and diversity gain with low implementation cost.

Recently, several precoding based conventional STBC systems have been investigated for massive MIMO scenarios in [18]-[24]. Specifically, noncoherent massive space-time block codes is designed for uplink communications in [18], while the massive STBC systems of [19]-[24] are designed for downlink communications. In [19], the authors proposed a novel massive OSTBC scheme by embedding smaller OSTBCs into massive configurations at the expense of degraded performance. Then, the authors of [20]-[22] proposed omnidirectional OSTBC and Q-OSTBC for massive MIMO systems, which attain the diversity gain by employing the precoding concept. Based on the same concept, the authors of [23] proposed fulldiversity STBC for massive MIMO. Finally, the authors of [24] presented the design criteria for the precoding based omnidirectional massive STBC systems.

However, the aforementioned schemes in [19]-[24] are designed for closed-loop massive MIMO downlink communication systems, which require the channel state information (CSI) at the transmitter and significantly increase the complexity. These massive STBC schemes also require numerous RF chains, resulting in substantial implementation cost and signal processing complexity. Furthermore, their common shortcoming is the low transmission rate. Therefore, it is meaningful to design a STBC scheme with high throughput for open-loop massive MIMO downlink communication systems.

With the development of massive MIMO, spatial modulation (SM), which implicitly transmits information relying on the activated antenna indices, has been considered as a promising low-cost single RF chain based open-loop massive MIMO candidate [25]-[30]. To further improve the diversity gain of SM, the STBC based SM (STBC-SM) scheme was proposed in [31]. Then, several variants of STBC-SM with high transmission rate and modified codeword were proposed in [32]-[35]. However, the common feature of these STBCSM schemes is that the information bits are transmitted as 
amplitude phase modulation (APM) symbols by one activated antenna combination (AC). In massive MIMO configurations, the selection process of $\mathrm{AC}$ becomes more complicated, which makes the bit-to-symbol mapping impractical. More importantly, the diversity gains of these schemes also become slight in the context of massive MIMO setup. In order to address the above issues, we proposed a novel STBC-SM in [36], where the SM symbols were taken as a whole vector to invoke for the Alamouti coding, which significantly simplified the AC design and the bit-to-symbol mapping. We refer to this scheme as Alamouti-SM in this paper. Moreover, it is demonstrated in [36] that Alamouti-SM outperforms STBC-SM in [31] in terms of bit error rate (BER). Non-coherent STBC-SM schemes were also developed in [37]-[41]. However, the above mentioned STBC-SM schemes were mainly designed for two activated TAs. To make STBC-SM applicable for large number of activated TAs, the authors of [42] proposed the spatially modulated orthogonal space-time block codes, where four RF chains were considered at the transmitter and double spacetime transmit diversity was achieved. However, it significantly complicated the space codeword design since the total number of space codewords was extremely large, i.e., $4^{N_{t}-2}$ at massive MIMO setups, where $N_{t}$ is the number of TAs. Then, we proposed a generalized STBC-SM (GSTBC-SM) system [43] to further exploit the benefits of the generalized STBC structure and SM scheme. However, it only considered low transmission rate based OSTBC-SM schemes and was only suitable for small-scale MIMO.

Against the above background, the contributions of this paper are summarized as follows.

1) We propose a novel GSTBC-SM structure for openloop massive MIMO downlink communication systems. In the proposed GSTBC-SM scheme, the information bits are partitioned into $K$ groups with each group modulated by SM, which will be taken as a node for conventional STBC coding (i.e OSTBC, Q-OSTBC). Hence the transmitter is capable of obtaining a flexible transmission rate and diversity order depending on the specific conventional STBC structure and the number of TAs. More specifically, both OSTBC-SM and QOSTBC-SM schemes are studied in the context of massive MIMO configurations.

2) We propose a low-complexity massage passing (MP) detector and a low-complexity block minimum mean square equalization (B-MMSE) detector for different GSTBC-SM systems, which are capable of approaching the maximum likelihood (ML) detector's performance with a significant complexity reduction. Furthermore, the proposed low-complexity detectors based GSTBCSM are capable of providing substantial performance gains over the conventional GSTBC, SM family [47] and zero-forcing (ZF) precoded MIMO [48] systems in the context of massive MIMO configurations.

3) Both the accurate and approximate average bit error probability (ABEP) are derived for our proposed GSTBC-SM scheme, which are verified by simulation results. Specifically, the performance of the proposed approximate ABEP approaches that of accurate counterpart with significantly reduced complexity.

The remainder of this paper is organized as follows. Section II gives a brief introduction of the conventional STBC schemes. Section III introduces the system model of the proposed GSTBC-SM. In Section IV, we propose low-complexity detectors for our GSTBC-SM schemes. In Section V, we derive the ABEP upper bound of the proposed GSTBC-SM scheme. Simulation results are presented in Section VI. Finally, Section VII concludes this paper.

Notation: $\|\cdot\|^{2}$ denotes the two norm of a matrix. $|\cdot|$ represents the magnitude of a complex quantity. $(\cdot)^{*},(\cdot)^{T}$ and $(\cdot)^{H}$ stand for the conjugate, transpose and the Hermitian transpose of a vector/matrix, respectively. $\mathbf{I}_{N}$ denotes $N \times N$ identity matrix. $\operatorname{Tr}(\mathbf{A})$ represents the trace of the matrix $\mathbf{A}$.

\section{AN OVER REVIEW OF EXISTING STBC SCHEMES}

For a conventional STBC-MIMO having $N_{t}^{c}$ TAs and $N_{r}$ receive antennas (RAs), $K$ APM symbols are transmitted over $T$ time slots by STBC coding, hence the coding ratio $R$ is defined as $R=K / T$. As mentioned in introduction, there are lots of STBC schemes having varied $R$. For simplicity, we mainly illustrate the GSTBC-SM system based on the OSTBC scheme with $K=3 R=0.75$ and the Q-OSTBC with $K=$ $4 R=1$ in this paper [1]. To ease understanding, we first introduce the two schemes as follows.

\section{A. OSTBC scheme with $K=3 R=3 / 4$}

For an OSTBC system having $N_{t}^{c}=4$ and $R=3 / 4$, the information bits are partitioned into $K=3$ groups, which are modulated into three $M$-APM symbols as $x_{1}, x_{2}$ and $x_{3}$. According to [1], the transmitted signal of OSTBC are expressed as

$$
\mathbf{G}_{434}=\left[\begin{array}{cccc}
x_{1} & -x_{2}^{*} & x_{3}^{*} & 0 \\
x_{2} & x_{1}^{*} & 0 & x_{3}^{*} \\
x_{3} & 0 & -x_{1}^{*} & -x_{2}^{*} \\
0 & x_{3} & x_{2} & -x_{1}
\end{array}\right]_{4 \times 4},
$$

where $\mathbf{G}_{434} \mathbf{G}_{434}^{H}=3 \mathbf{I}_{4}$.

\section{B. $Q$-OSTBC scheme with $K=4 R=1$}

For a Q-OSTBC scheme with $R=1$, the information bits are partitioned into four groups, which are modulated into four $M$-APM symbols as $x_{1}, x_{2}, x_{3}$ and $x_{4}$. According to [1], the transmitted signal of Q-OSTBC are expressed as

$$
\mathbf{G}_{Q 1}=\left[\begin{array}{cccc}
x_{1} & -x_{2}^{*} & -x_{3}^{*} e^{-j \phi} & x_{4} e^{j \phi} \\
x_{2} & x_{1}^{*} & -x_{4}^{*} e^{-j \phi} & -x_{3} e^{j \phi} \\
x_{3} e^{j \phi} & -x_{4}^{*} e^{-j \phi} & x_{1}^{*} & -x_{2} \\
x_{4} e^{j \phi} & x_{3}^{*} e^{-j \phi} & x_{2}^{*} & x_{1}
\end{array}\right] .
$$

Other generalized STBC schemes can be found in [5]-[14]. 


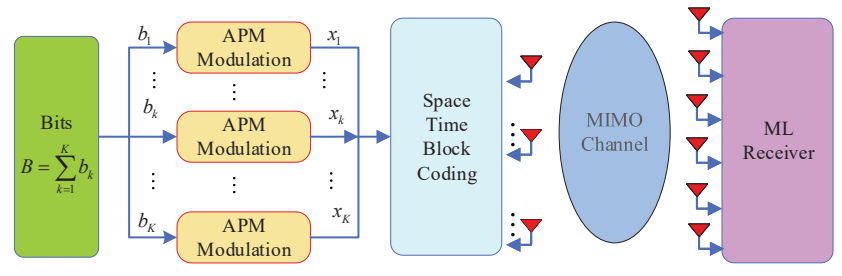

(a) Conventional GSTBC system

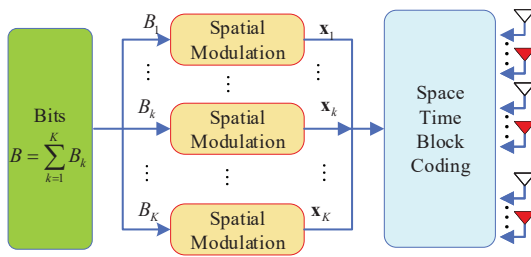

(b) Proposed GSTBC-SM system

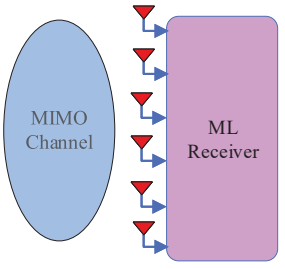

Fig. 1. System models of the conventional GSTBC and the proposed GSTBCSM schemes.

\section{Proposed Generalized Space Time Block Coding SPATIAL Modulation}

We consider a GSTBC-SM structure with $N_{t}$ TAs and $N_{r}$ receive antennas over flat Rayleigh fading channels via $T$ time slots. Fig. 1 presents the system model of GSTBC$\mathrm{SM}$, where the information bits with length of $B$ are split into $K$ blocks to map $K \mathrm{SM}$ symbols and then conventional GSTBC structure is invoked for these symbols. The coding ratio is $R=K / T$, which is the same as the conventional counterpart. Explicitly, for the $k$-th block information bits with length of $B_{k}=\left\lceil\log _{2} n_{t}\right\rceil+M_{k}$ are further partitioned into two parts to map an SM symbol $\mathbf{x}_{k}$ using $n_{t}$ TAs and $M_{k}$-APM modulation, where $\left\lceil\log _{2} x\right\rceil$ denotes the ceil operator. If $n_{t}$ is a power of 2, the SM mapping method of [25] is employed. Otherwise, $\mathbf{x}_{k}$ is obtained by the mapping method in [36]. Then, the $K$ SM symbols are invoked into a specific STBC structure [1]. Hence, the transmission rate of the proposed GSTBC-SM is

$$
R_{p}=\frac{\sum_{k=1}^{K}\left\lceil\log _{2} n_{t}\right\rceil+M_{k}}{T} .
$$

According to [1], there are numerous STBC codes with different $R$. In the proposed GSTBC-SM, the value of $n_{t}$ is selected based on the specific STBC structure and the number of TAs $N_{t}$. Hence the throughput is determined by the specific STBC structure and the value of $N_{t}$.

Next, our proposed GSTBC-SM structure with specific examples are introduced and its generalization design will be developed.

\section{A. Proposed OSTBC-SM with $K=3$ and $R=3 / 4$}

\section{1) Transmit structure:}

In this subsection, GSTBC-SM with OSTBC structure is introduced. For an OSTBC-SM scheme with $R=3 / 4$, the information bits are partitioned into three groups, which are modulated into three SM symbols using $n_{t}=N_{t} / 4$ TAs as

$$
\mathbf{x}_{k}=(\underbrace{0, \ldots, 0}_{q_{k}-1}, s_{k}, \underbrace{0, \ldots, 0}_{N_{t} / 4-q_{k}}), k=1,2,3,
$$

where $q_{k}$ is the activated TA index in $\mathbf{x}_{k}$ and $s_{k}$ belongs to the constellation symbol set $\mathbb{M}$. According to (1), the transmitted signal $\mathbf{S}_{3 / 4} \in \mathbb{C}^{N_{t} \times 4}$ of the OSTBC-SM scheme can be obtained via (1) using $\mathbf{x}_{1}, \mathbf{x}_{2}$ and $\mathbf{x}_{3}$ as

$$
\mathbf{S}_{3 / 4}=\left[\begin{array}{cccc}
\mathbf{x}_{1} & -\mathbf{x}_{2}^{*} & \mathbf{x}_{3}^{*} & \mathbf{O}_{N_{t} / 4} \\
\mathbf{x}_{2} & \mathbf{x}_{1}^{*} & \mathbf{O}_{N_{t} / 4} & \mathbf{x}_{3}^{*} \\
\mathbf{x}_{3} & \mathbf{O}_{N_{t} / 4} & -\mathbf{x}_{1}^{*} & -\mathbf{x}_{2}^{*} \\
\mathbf{O}_{N_{t} / 4} & \mathbf{x}_{3} & \mathbf{x}_{2} & -\mathbf{x}_{1}
\end{array}\right]_{N_{t} \times 4} .
$$

2) Diversity order and coding gain analysis:

In this subsection, the diversity and coding gain of the proposed OSTBC-SM with $R=3 / 4$ are analyzed. Assume that $\mathbf{S}_{3 / 4}^{i}$ and $\mathbf{S}_{3 / 4}^{j}$ are two different OSTBC-SM symbols as

$$
\mathbf{S}_{3 / 4}^{i}=\left[\begin{array}{cccc}
\mathbf{x}_{1}^{i}-\mathbf{x}_{2}^{i *} & \mathbf{x}_{3}^{i *} & 0 \\
\mathbf{x}_{2}^{i} & \mathbf{x}_{1}^{i *} & 0 & \mathbf{x}_{3}^{i *} \\
\mathbf{x}_{3}^{i} & 0 & -\mathbf{x}_{1}^{i *}-\mathbf{x}_{2}^{i *} \\
0 & \mathbf{x}_{3}^{i} & \mathbf{x}_{2}^{i} & -\mathbf{x}_{1}^{i}
\end{array}\right], \mathbf{S}_{3 / 4}^{j}=\left[\begin{array}{cccc}
\mathbf{x}_{1}^{j}-\mathbf{x}_{2}^{j *} & \mathbf{x}_{3}^{j *} & 0 \\
\mathbf{x}_{2}^{j *} & \mathbf{x}_{1}^{j *} & 0 & \mathbf{x}_{3}^{j *} \\
\mathbf{x}_{3}^{j} & 0 & -\mathbf{x}_{1}^{j *}-\mathbf{x}_{2}^{j *} \\
0 & \mathbf{x}_{3}^{j} & \mathbf{x}_{2}^{j} & -\mathbf{x}_{1}^{j}
\end{array}\right],
$$

the coding gain of the OSTBC-SM scheme is defined as

$$
G_{3 / 4}=\min _{\mathbf{S}_{3 / 4}^{i} \neq \mathbf{S}_{3 / 4}^{j}}\left|\operatorname{det}\left(\Delta_{3 / 4}^{H} \Delta_{3 / 4}\right)\right|,
$$

where $\Delta_{3 / 4}=\mathbf{S}_{3 / 4}^{i}-\mathbf{S}_{3 / 4}^{j}$. The value of $\Delta_{3 / 4}^{H} \Delta_{3 / 4}$ can be further obtained as

$$
\Delta_{3 / 4}^{H} \Delta_{3 / 4}=\left[\begin{array}{cccc}
A_{3 / 4} & 0 & 0 & 0 \\
0 & A_{3 / 4} & 0 & 0 \\
0 & 0 & A_{3 / 4} & 0 \\
0 & 0 & 0 & A_{3 / 4}
\end{array}\right]
$$

where $A_{3 / 4}=\left\|\mathbf{x}_{1}^{i}-\mathbf{x}_{1}^{j}\right\|^{2}+\left\|\mathbf{x}_{2}^{i}-\mathbf{x}_{2}^{j}\right\|^{2}+\left\|\mathbf{x}_{3}^{i}-\mathbf{x}_{3}^{j}\right\|^{2}$. Moreover, the value of $A_{3 / 4}$ can be further obtained as

$A_{3 / 4}=\left\{\begin{array}{l}0, \text { if } \mathbf{x}_{k}^{i}=\mathbf{x}_{k}^{j}, k=1,2,3 ; \\ \left\|\mathbf{x}_{k}^{i}-\mathbf{x}_{k}^{j}\right\|^{2}, \text { if } \mathbf{x}_{k}^{i}=\mathbf{x}_{k}^{j}, k=2,3 ; \\ \left\|\mathbf{x}_{1}^{i}-\mathbf{x}_{1}^{j}\right\|^{2}+\left\|\mathbf{x}_{2}^{i}-\mathbf{x}_{2}^{j}\right\|^{2}, \text { if } \mathbf{x}_{k}^{i}=\mathbf{x}_{k}^{j}, k=3 ; \\ \left\|\mathbf{x}_{1}^{i}-\mathbf{x}_{1}^{j}\right\|^{2}+\left\|\mathbf{x}_{2}^{i}-\mathbf{x}_{2}^{j}\right\|^{2}+\left\|\mathbf{x}_{3}^{i}-\mathbf{x}_{3}^{j}\right\|^{2} \text { if } \mathbf{x}_{l}^{i} \neq \mathbf{x}_{l}^{j}, \forall l .\end{array}\right.$

It can be seen from (8) that the rank of $\Delta_{3 / 4}^{H} \Delta_{3 / 4}$ is four, so that the diversity order of the OSTBC-SM is four. According to (7)-(9), its coding gain can be finally obtained as

$$
G_{3 / 4}=\min _{\mathbf{S}_{3 / 4}^{i} \neq \mathbf{S}_{3 / 4}^{j}} A_{3 / 4}^{4}=\min _{s_{i} \neq s_{j} \in \mathbb{M}}\left[\left|s_{i}-s_{j}\right|^{8},\left(\left|s_{i}\right|^{2}+\left|s_{j}\right|^{2}\right)^{4}\right] .
$$

\section{B. Proposed $Q$-OSTBC-SM scheme with $K=4$ and $R=1$}

1) Transmit structure:

In this subsection, GSTBC-SM with Q-OSTBC structure is introduced. For an Q-OSTBC-SM scheme associated with $R=1$, the information bits are split into four groups, which 
are modulated into four SM symbols using $N_{t} / 4$ TAs by (4). The transmitted signal $\mathbf{S}_{Q 1} \in \mathbb{C}^{N_{t} \times 4}$ of the Q-OSTBC-SM scheme can be obtained via (2) using four SM symbols $\mathbf{x}_{1}$, $\mathbf{x}_{2}, \mathbf{x}_{3}$ and $\mathbf{x}_{4}$ as

$$
\mathbf{S}_{Q 1}=\left[\begin{array}{cccc}
\mathbf{x}_{1} & -\mathbf{x}_{2}^{*} & -\mathbf{x}_{3}^{*} e^{-j \phi} & \mathbf{x}_{4} e^{j \phi} \\
\mathbf{x}_{2} & \mathbf{x}_{1}^{*} & -\mathbf{x}_{4}^{*} e^{-j \phi} & -\mathbf{x}_{3} e^{j \phi} \\
\mathbf{x}_{3} e^{j \phi}-\mathbf{x}_{4}^{*} e^{-j \phi} & \mathbf{x}_{1}^{*} & -\mathbf{x}_{2} \\
\mathbf{x}_{4} e^{j \phi} & \mathbf{x}_{3}^{*} e^{-j \phi} & \mathbf{x}_{2}^{*} & \mathbf{x}_{1}
\end{array}\right]
$$

2) Diversity order and coding gain analysis:

Assuming that $\mathbf{S}_{Q 1}^{i}$ and $\mathbf{S}_{Q 1}^{j}$ are mapping by (11) using $\left(\mathbf{x}_{1}^{i}, \mathbf{x}_{2}^{i}, \mathbf{x}_{3}^{i}, \mathbf{x}_{4}^{i}\right)$ and $\left(\mathbf{x}_{1}^{j}, \mathbf{x}_{2}^{j}, \mathbf{x}_{3}^{j}, \mathbf{x}_{4}^{j}\right)$, respectively, the coding gain can be obtained as

$$
G_{Q}=\min _{\mathbf{S}_{Q 1}^{i} \neq \mathbf{S}_{Q 1}^{j}}\left|\operatorname{det}\left(\Delta_{Q 1}^{H} \Delta_{Q 1}\right)\right|,
$$

where $\Delta_{Q 1}=\mathbf{S}_{Q 1}^{i}-\mathbf{S}_{Q 1}^{j}$ is expressed as

$$
\begin{aligned}
& \Delta_{Q 1}= \\
& {\left[\begin{array}{cccc}
\mathbf{x}_{1}^{i}-\mathbf{x}_{1}^{j} & \left(\mathbf{x}_{2}^{j}\right)^{*}-\left(\mathbf{x}_{2}^{i}\right)^{*} & {\left[\left(\mathbf{x}_{3}^{j}\right)^{*}-\left(\mathbf{x}_{3}^{i}\right)^{*}\right] e^{-j \phi}\left(\mathbf{x}_{4}^{i}-\mathbf{x}_{4}^{j}\right) e^{j \phi}} \\
\mathbf{x}_{2}^{i}-\mathbf{x}_{2}^{j} & \left(\mathbf{x}_{1}^{i}\right)^{*}-\left(\mathbf{x}_{1}^{j}\right)^{*} & {\left[\left(\mathbf{x}_{4}^{j}\right)^{*}-\left(\mathbf{x}_{4}^{i}\right)^{*}\right] e^{-j \phi}\left(\mathbf{x}_{3}^{j}-\mathbf{x}_{3}^{i}\right) e^{j \phi}} \\
\left(\mathbf{x}_{3}^{i}-\mathbf{x}_{3}^{j}\right) e^{j \phi}\left[\left(\mathbf{x}_{4}^{j}\right)^{*}-\left(\mathbf{x}_{4}^{i}\right)^{*}\right] e^{-j \phi} & \left(\mathbf{x}_{1}^{i}\right)^{*}-\left(\mathbf{x}_{1}^{j}\right)^{*} & \mathbf{x}_{2}^{j}-\mathbf{x}_{2}^{i} \\
\left(\mathbf{x}_{4}^{i}-\mathbf{x}_{4}^{j}\right) e^{j \phi}\left[\left(\mathbf{x}_{3}^{i}\right)^{*}-\left(\mathbf{x}_{3}^{j}\right)^{*}\right] e^{-j \phi} & \left(\mathbf{x}_{2}^{i}\right)^{*}-\left(\mathbf{x}_{2}^{j}\right)^{*} & \mathbf{x}_{1}^{i}-\mathbf{x}_{1}^{j}
\end{array}\right]}
\end{aligned}
$$

According to (13), the value of $\Delta_{Q 1}^{H} \Delta_{Q 1}$ can be obtained by

$$
\Delta_{Q 1}^{H} \Delta_{Q 1}=\left[\begin{array}{cccc}
A_{Q 1} & 0 & 0 & B_{Q 1} \\
0 & A_{Q 1} B_{Q 1} & 0 \\
0 & B_{Q 1} & A_{Q 1} & 0 \\
B_{Q 1} & 0 & 0 & A_{Q 1}
\end{array}\right],
$$

where the value of $A_{Q 1}$ and $B_{Q 1}$ can be calculated as

$$
\begin{aligned}
& A_{Q 1}=\left\|\boldsymbol{\Lambda}_{1}\right\|^{2}+\left\|\boldsymbol{\Lambda}_{2}\right\|^{2}+\left\|\boldsymbol{\Lambda}_{3}\right\|^{2}+\left\|\boldsymbol{\Lambda}_{4}\right\|^{2}, \\
& B_{Q 1}=2 \Re\left[\left(\boldsymbol{\Lambda}_{1}\right)^{H} \boldsymbol{\Lambda}_{4} e^{j \phi}-\left(\boldsymbol{\Lambda}_{2}\right)^{H} \boldsymbol{\Lambda}_{3} e^{j \phi}\right],
\end{aligned}
$$

where $\boldsymbol{\Lambda}_{\mathbf{1}}=\mathbf{x}_{1}^{i}-\mathbf{x}_{1}^{j} \in \varphi, \boldsymbol{\Lambda}_{\mathbf{2}}=\mathbf{x}_{2}^{i}-\mathbf{x}_{2}^{j} \in \varphi, \boldsymbol{\Lambda}_{\mathbf{3}}=\mathbf{x}_{3}^{i}-\mathbf{x}_{3}^{j} \in$ $\varphi, \boldsymbol{\Lambda}_{\mathbf{4}}=\mathbf{x}_{4}^{i}-\mathbf{x}_{4}^{j} \in \varphi$. Explicitly, the value of $\varphi$ is defined by $\varphi=\left\{\left[\cdots, s_{i}-s_{j} \cdots\right]^{T},\left[\cdots, s_{i}, \cdots,-s_{j} \cdots\right]^{T}\right\}, s_{i}, s_{j} \in \mathbb{M}$.

According to [1], we have

$$
\operatorname{det}\left(\begin{array}{l}
A B \\
C D
\end{array}\right)=\operatorname{det}(A) \operatorname{det}\left(D-C A^{-1} B\right) .
$$

Hence, the value of $\operatorname{det}\left(\Delta_{Q 1}^{H} \Delta_{Q 1}\right)$ can be obtained via (14) and (17) as

$$
\begin{aligned}
& \operatorname{det}\left(\Delta_{Q 1}^{H} \Delta_{Q 1}\right)=\left(A_{Q 1}+B_{Q 1}\right)^{2}\left(A_{Q 1}-B_{Q 1}\right)^{2} \\
& =\left\{\begin{array}{l}
\left\|\boldsymbol{\Lambda}_{1}\right\|^{8}, \text { if } \boldsymbol{\Lambda}_{k}=\mathbf{O}_{N_{t} / 4}, k=2,3,4, \\
\left\|\boldsymbol{\Lambda}_{1}\right\|^{8}\left|1-e^{j 2 \phi}\right|^{4}, \text { if } \boldsymbol{\Lambda}_{k}=\mathbf{O}_{N_{t} / 4}, k=2,3, \boldsymbol{\Lambda}_{1}=\boldsymbol{\Lambda}_{4}, \\
{\left[\left(\left\|\boldsymbol{\Lambda}_{1}-\boldsymbol{\Lambda}_{4} e^{j \phi}\right\|^{2}+\left\|\boldsymbol{\Lambda}_{2}+\boldsymbol{\Lambda}_{3} e^{j \phi}\right\|^{2}\right)^{2}\right.} \\
\left.\times\left(\boldsymbol{\Lambda}_{1}+\boldsymbol{\Lambda}_{4} e^{j \phi 2}+\boldsymbol{\Lambda}_{2}-\boldsymbol{\Lambda}_{3} e^{j \phi 2}\right)^{2}\right], \text { else. }
\end{array}\right.
\end{aligned}
$$

Then, the coding gain can be finally obtained as

$$
\begin{aligned}
& G_{Q 1}=\min _{\mathbf{S}_{Q 1}^{i} \neq \mathbf{S}_{Q 1}^{j}}\left|\operatorname{det}\left(\Delta_{Q 1}^{H} \Delta_{Q 1}\right)\right| \\
& =\min _{s_{i} \neq s_{j} \in M}\left[\left|\left(s_{i}-s_{j}\right)\right|^{8}\left|\left(1-e^{j 2 \phi}\right)\right|^{4},\left|\left(s_{i}-s_{j}\right)\right|^{8}\right] .
\end{aligned}
$$

TABLE I

MAXIMUM CODING GAIN COMPARISONS BETWEEN THE PROPOSED GSTBC-SM SCHEMES AND CONVENTIONAL STBC SCHEMES

\begin{tabular}{|c|c|c|c|c|}
\hline \hline \multicolumn{5}{|c|}{ Rate=3 bpcu } \\
\hline Scheme & OSTBC & OSTBC-SM & Q-OSTBC \\
$\left(N_{t}, K, M\right)$ & $(4,3,16)$ & $(8,3,8)$ & $(4,4,8)$ & $\begin{array}{c}\text { Q-OSTBC-SM } \\
(8,4,4)\end{array}$ \\
\hline Coding gain & $5.4 \mathrm{e}-4$ & 0.1177 & 0.1177 & 16 \\
\hline \hline \multicolumn{5}{|c|}{ Rate=6 bpcu } \\
\hline Scheme & OSTBC & OSTBC-SM & Q-OSTBC & Q-OSTBC-SM \\
$\left(N_{t}, K, M\right)$ & $(4,3,256)$ & $(64,3,16)$ & $(4,4,64)$ & $(64,4,4)$ \\
\hline Coding gain & $1.3 \mathrm{e}-13$ & $5.4 \mathrm{e}-4$ & $8.6 \mathrm{e}-9$ & 16 \\
\hline
\end{tabular}

As a result, the diversity order of Q-OSTBC-SM is four and the optimal $\phi$ can be obtained as

$$
\begin{aligned}
& \phi=\max _{\phi \in(0, \pi)}\left(G_{Q 1}\right)=\max _{\phi \in(0, \pi)}\left(\left|1-e^{j 2 \phi}\right|^{4}, 1\right) \\
& =\max _{\phi \in(0, \pi)}\left(\left|1-e^{j 2 \phi}\right|^{4}, 1\right) \\
& =\max _{\phi \in(0, \pi)}\left(|2-2 \cos (2 \phi)|^{2}, 1\right) \\
& =\max _{\phi \in(0, \pi)}\left[4\left(2 \sin ^{2}(\phi)\right)^{2}, 1\right] .
\end{aligned}
$$

According to (20), the range of $\phi$ is calculated as

$$
4\left(2 \sin ^{2}(\phi)\right)^{2} \geq 1 \Rightarrow \pi / 6 \leq \phi \leq 5 \pi / 6 .
$$

The optimal coding gain of Q-OSTBC-SM is expressed as

$$
G_{Q 1}^{\text {opt. }}=\left|\left(s_{i}-s_{j}\right)\right|^{8} .
$$

In order to provide further insights, Table I compares the maximum coding gains of the proposed OSTBC-SM and QOSTBC-SM systems to those of conventional counterpart with the identical number of RF chains and throughput, where bpcu denotes the bits per channel use. It is observed from Table I that the proposed system provides significant coding gain over the corresponding STBC counterpart. As $N_{t}$ increases, this superiority becomes more apparent.

\section{Generalized design}

According to [1]-[15], there are numerous eminent STBC schemes, which can be straightforward to extend into our proposed GSTBC-SM structure. The generalize design of GSTBC-SM can be detailed as follows.

Step 1: Obtain a conventional GSTBC structure by [1]-[15];

Step 2: Obtain the setups $\left(N_{t}^{c}, K, T\right)$ of the conventional GSTBC scheme;

Step 3: Obtain the setups $\left(n_{t}, K, T\right)$ of the proposed GSTBC-SM scheme based on the values of $\left(N_{t}^{c}, K, T\right)$ and $N_{t}$;

Step 4: Obtain the APM modulation order $M_{k}$ for each SM symbol based on the given throughput of (3).

Step 5: Obtain $K$ SM symbols using $n_{t}$ and $M_{k}$;

Step 6: Obtain the proposed GSTBC-SM symbol by substituting $K$ SM symbols into an APM symbol of conventional STBC structure obtained by Step 1;

Step 7: Analyze the diversity order and coding gain of the proposed GSTBC-SM system. For an OSTBC-SM system, the analysis can be carried out by the approach of (7)-(8), while that can be implemented by the method of (14)-(19) for a Q-OSTBC-SM system. 


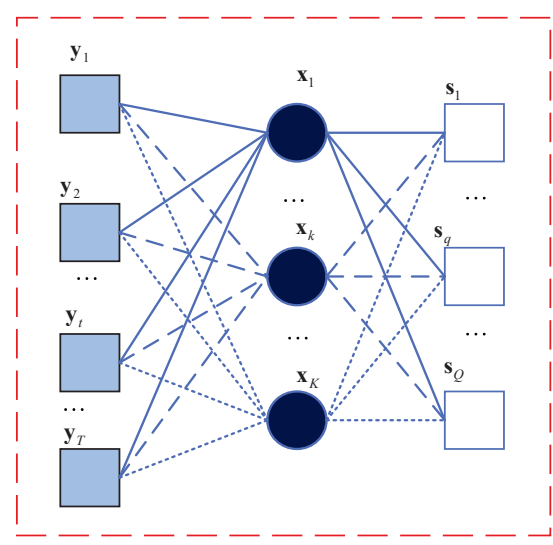

(a) Factor graph

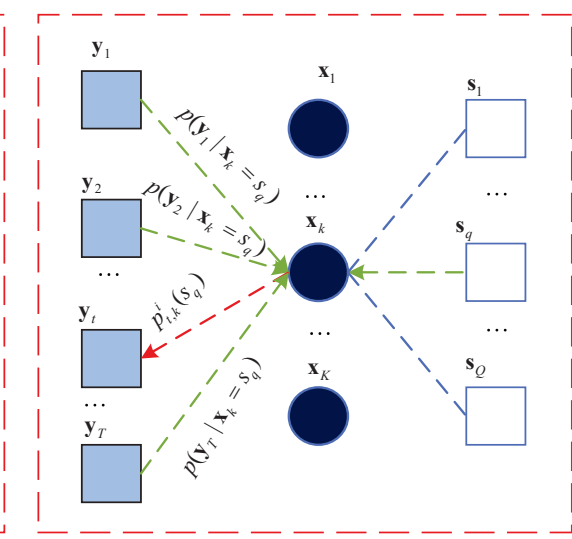

(b) Message from $\mathbf{x}_{k}$ to $\mathbf{y}_{t}$

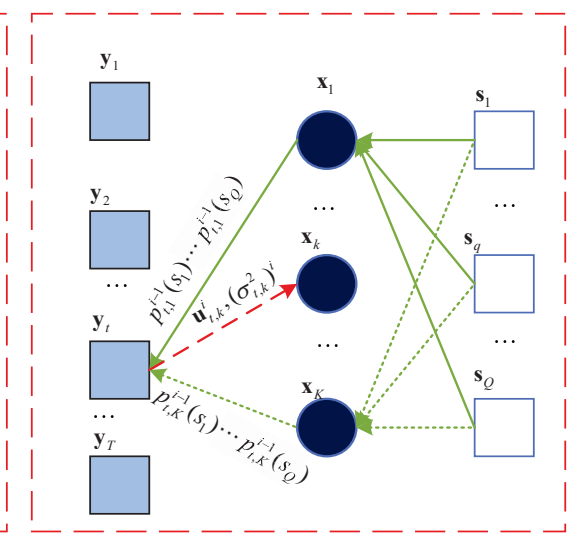

(c) Message from $\mathbf{y}_{t}$ to $\mathbf{x}_{k}$

Fig. 2. Factor graph of the proposed MP detector.

Step 8: Perform signal detection for the proposed GSTBCSM system.

\section{Optimal detector}

In this subsection, the ML detector for our proposed GSTBC-SM is designed. Specifically, according to (5) and (11), the received signal $\mathbf{Y} \in \mathbb{C}^{N_{r} \times T}$ can be formulated as

$$
\mathbf{Y}=\mathbf{H S}+\mathbf{N},
$$

where $\mathbf{H} \in \mathbb{C}^{N_{r} \times N_{t}}$ and $\mathbf{N} \in \mathbb{C}^{N_{r} \times T}$ represent the channel and noise matrices, whose elements obey the complex Gaussian distributions associated with $\mathcal{C N}(0,1)$ and $\mathcal{C N}\left(0, \sigma^{2}\right)$, respectively, where $\sigma^{2}$ is the noise variance, and $\mathbf{S} \in \mathbb{C}^{N_{t} \times T}$ denotes the GSTBC-SM symbol. Then, the optimal ML detector can be formulated as

$$
\hat{\mathbf{S}}=\underset{\mathbf{S} \in \mathbb{S}}{\arg \min }\|\mathbf{Y}-\mathbf{H S}\|^{2},
$$

where $\mathbb{S}$ is the set of the proposed GSTBC-SM symbols. According to (3), it is obvious that the complexity order is $O\left(2^{R_{p} T}\right)$. Hence the complexity of the ML detector will become extremely high in the context of massive MIMO configurations. Low-complexity detectors become important for our proposed GSTBC-SM in massive MIMO communication scenarios, which will be introduced in the following section.

\section{LOW-COMPLEXITY DETECTORS DESIGN FOR THE PROPOSED GSTBC-SM SYSTEMS}

In this section, low-complexity MP and B-MMSE detectors are designed for our GSTBC-SM systems based on the specific STBC structures. Both of them are capable of approaching the optimal ML detector with significantly reduced complexity.

\section{A. Proposed MP detector}

Fig. 2 presents the factor graph of the proposed MP detector. As observed from Fig. 2, the messages are passing between the observation node $\mathbf{y}_{t}$ to the variable node $\mathbf{x}_{k}$, which can be expressed by [45]

$$
\begin{aligned}
& V_{\mathbf{y}_{t} \rightarrow \mathbf{x}_{k}}^{i}=\left\{\begin{array}{l}
\mathbf{u}_{t, k}^{i}=\sum_{v \neq k} \mathbf{H}_{(v)} \sum_{q=1}^{Q} \mathbf{s}_{q} p_{t v}^{i-1}\left(\mathbf{s}_{q}\right) \\
\left(\sigma_{t, k}^{2}\right)^{i}=\sum_{v \neq k}\left(\sum_{q=1}^{Q}\left\|\mathbf{H}_{(v)} \mathbf{s}_{q}\right\|^{2} p_{t v}^{i-1}\left(\mathbf{s}_{q}\right)\right. \\
\left.-\left\|\mathbf{H}_{(v)} \sum_{q=1}^{Q} \mathbf{s}_{q} p_{t v}^{i-1}\left(\mathbf{s}_{q}\right)\right\|^{2}\right)
\end{array}\right. \\
& V_{\mathbf{y}_{t} \leftarrow \mathbf{x}_{k}}^{i}=p_{t k}^{i}\left(\mathbf{s}_{q}\right)=\prod_{j=1, j \neq t}^{T} p\left(\mathbf{y}_{j} \mid \mathbf{x}_{k}=\mathbf{s}_{q}\right),
\end{aligned}
$$

where $\mathbf{u}_{t, k}^{i}$ and $\left(\sigma_{t, k}^{2}\right)^{i}$ denote the mean value and variance associated with the received signal $\mathbf{y}_{t}$ and transmit signal $\mathbf{x}_{k}$, respectively, $\mathbf{H}_{(k)}$ is the channel matrix associated with $\mathbf{x}_{k}$, $\mathbf{s}_{q}$ is the possible SM symbol, $p_{t v}^{i-1}\left(\mathbf{s}_{q}\right)$ is the probability of $\mathbf{s}_{q}$ associated with $\mathbf{y}_{t}$ and $\mathbf{x}_{k}$ in the $(i-1)$-th iteration calculation. $V_{\mathbf{y}_{t} \rightarrow \mathbf{x}_{k}}^{i}$ denotes the message from the observation node $\mathbf{y}_{t}$ to the variable node $\mathbf{x}_{k}$, while $V_{\mathbf{y}_{t} \leftarrow \mathbf{x}_{k}}^{i}$ denotes the message from the variable node $\mathbf{x}_{k}$ to the observation node $\mathbf{y}_{t}$. According to (25), in the proposed MP detector, the calculation of $\mathbf{u}_{t, k}^{i},\left(\sigma_{t, k}^{2}\right)^{i}$ and $p_{t v}^{i-1}\left(\mathbf{s}_{q}\right)$ are the key factors. Next, we will introduce these calculations by specific examples.

1) $M P$ detector for OSTBC-SM with $R=3 / 4$ :

To obtain the values of $\mathbf{u}_{t, k}^{i},\left(\sigma_{t, k}^{2}\right)^{i}$ and $p_{t v}^{i-1}\left(\mathbf{s}_{q}\right)$, we first obtain the factor graph by the received signal of (23). Specifically, assuming that $\mathbf{Y}=\left[\mathbf{y}_{1}, \mathbf{y}_{2}, \mathbf{y}_{3}, \mathbf{y}_{4}\right], \mathbf{H}=$ $\left[\mathbf{H}_{1} \mathbf{H}_{2} \mathbf{H}_{3} \mathbf{H}_{4}\right]$, and $\mathbf{N}=\left[\mathbf{n}_{1}, \mathbf{n}_{2}, \mathbf{n}_{3}, \mathbf{n}_{4}\right]$, the received signal can be rewritten as

$$
\begin{aligned}
& \mathbf{y}_{1}=\mathbf{H}_{1} \mathbf{x}_{1}+\underbrace{\mathbf{H}_{2} \mathbf{x}_{2}+\mathbf{H}_{3} \mathbf{x}_{3}+\mathbf{n}_{1}}_{\mathbf{w}_{1,1}} \\
& \mathbf{y}_{2}=\mathbf{H}_{2} \mathbf{x}_{1}^{*} \underbrace{-\mathbf{H}_{1} \mathbf{x}_{2}^{*}+\mathbf{H}_{4} \mathbf{x}_{3}+\mathbf{n}_{2}}_{\mathbf{w}_{2,1}} \\
& \mathbf{y}_{3}=-\mathbf{H}_{3} \mathbf{x}_{1}^{*}+\underbrace{\mathbf{H}_{1} \mathbf{x}_{3}^{*}+\mathbf{H}_{4} \mathbf{x}_{2}+\mathbf{n}_{3}}_{\mathbf{w}_{3,1}} \\
& \mathbf{y}_{4}=-\mathbf{H}_{4} \mathbf{x}_{1}+\underbrace{\mathbf{H}_{2} \mathbf{x}_{3}^{*}-\mathbf{H}_{3} \mathbf{x}_{2}^{*}+\mathbf{n}_{4}}_{\mathbf{w}_{4,1}},
\end{aligned}
$$




$$
\begin{aligned}
& \mathbf{y}_{1}=\mathbf{H}_{2} \mathbf{x}_{2}+\underbrace{\mathbf{H}_{1} \mathbf{x}_{1}+\mathbf{H}_{3} \mathbf{x}_{3}+\mathbf{n}_{1}}_{\mathbf{w}_{1,2}} \\
& \mathbf{y}_{2}=-\mathbf{H}_{1} \mathbf{x}_{2}^{*}+\underbrace{\mathbf{H}_{2} \mathbf{x}_{1}^{*}+\mathbf{H}_{4} \mathbf{x}_{3}+\mathbf{n}_{2}}_{\mathbf{w}_{2,2}} \\
& \mathbf{y}_{3}=\mathbf{H}_{4} \mathbf{x}_{2} \underbrace{-\mathbf{H}_{3} \mathbf{x}_{1}^{*}+\mathbf{H}_{1} \mathbf{x}_{3}^{*}+\mathbf{n}_{3}}_{\mathbf{w}_{3,2}} \\
& \mathbf{y}_{4}=-\mathbf{H}_{3} \mathbf{x}_{2}^{*} \underbrace{-\mathbf{H}_{4} \mathbf{x}_{1}+\mathbf{H}_{2} \mathbf{x}_{3}^{*}+\mathbf{n}_{4}}_{\mathbf{w}_{4,2}},
\end{aligned}
$$

and

$$
\begin{aligned}
& \mathbf{y}_{1}=\mathbf{H}_{3} \mathbf{x}_{3}+\underbrace{\mathbf{H}_{1} \mathbf{x}_{1}+\mathbf{H}_{2} \mathbf{x}_{2}+\mathbf{n}_{1}}_{\mathbf{w}_{1,3}} \\
& \mathbf{y}_{2}=\mathbf{H}_{4} \mathbf{x}_{3}+\underbrace{\mathbf{H}_{2} \mathbf{x}_{1}^{*}-\mathbf{H}_{1} \mathbf{x}_{2}^{*}+\mathbf{n}_{2}}_{\mathbf{w}_{2,3}} \\
& \mathbf{y}_{3}=\mathbf{H}_{1} \mathbf{x}_{3}^{*} \underbrace{-\mathbf{H}_{3} \mathbf{x}_{1}^{*}+\mathbf{H}_{4} \mathbf{x}_{2}+\mathbf{n}_{3}}_{\mathbf{w}_{3,3}} \\
& \mathbf{y}_{4}=\mathbf{H}_{2} \mathbf{x}_{3}^{*} \underbrace{-\mathbf{H}_{4} \mathbf{x}_{1}-\mathbf{H}_{3} \mathbf{x}_{2}^{*}+\mathbf{n}_{4}}_{\mathbf{w}_{4,3}},
\end{aligned}
$$

where $\mathbf{w}_{t, k}$ with $t=(1,2,3,4)$ and $k=(1,2,3)$ denotes the interference from symbols $\mathbf{x}_{v}$ with $v \neq k$. Moreover, $\mathbf{u}_{t, k}^{i}$ and $\left(\sigma_{t, k}^{2}\right)^{i}$ denote the mean value and variance of $\mathbf{w}_{t, k}$ at the $i$-th iteration, which are calculated in the Appendix A, and $p\left(\mathbf{y}_{j} \mid \mathbf{x}_{k}=s_{q}\right)$ denotes the conditional probability, which can be obtained by

$$
\begin{aligned}
& p\left(\mathbf{y}_{1} \mid \mathbf{x}_{1}\right) \propto \exp \left(\frac{-\left\|\mathbf{y}_{1}-\mathbf{H}_{1} \mathbf{x}_{1}-\mathbf{u}_{1,1}\right\|^{2}}{2 \sigma_{1,1}^{2}}\right), \\
& p\left(\mathbf{y}_{2} \mid \mathbf{x}_{1}\right) \propto \exp \left(\frac{-\left\|\mathbf{y}_{2}-\mathbf{H}_{2}\left(\mathbf{x}_{1}\right)^{*}-\mathbf{u}_{2,1}\right\|^{2}}{2 \sigma_{2,1}^{2}}\right), \\
& p\left(\mathbf{y}_{3} \mid \mathbf{x}_{1}\right) \propto \exp \left(\frac{-\left\|\mathbf{y}_{3}+\mathbf{H}_{3}\left(\mathbf{x}_{1}\right)^{*}-\mathbf{u}_{3,1}\right\|^{2}}{2 \sigma_{3,1}^{2}}\right) \\
& p\left(\mathbf{y}_{4} \mid \mathbf{x}_{1}\right) \propto \exp \left(\frac{-\left\|\mathbf{y}_{4}+\mathbf{H}_{4} \mathbf{x}_{1}-\mathbf{u}_{4,1}\right\|^{2}}{2 \sigma_{4,1}^{2}}\right), \\
& p\left(\mathbf{y}_{1} \mid \mathbf{x}_{2}\right) \propto \exp \left(\frac{-\left\|\mathbf{y}_{1}-\mathbf{H}_{2} \mathbf{x}_{2}-\mathbf{u}_{1,2}\right\|^{2}}{2 \sigma_{1,2}^{2}}\right), \\
& p\left(\mathbf{y}_{2} \mid \mathbf{x}_{2}\right) \propto \exp \left(\frac{-\left\|\mathbf{y}_{2}+\mathbf{H}_{1}\left(\mathbf{x}_{2}\right)^{*}-\mathbf{u}_{2,2}\right\|^{2}}{2 \sigma_{2,2}^{2}}\right), \\
& p\left(\mathbf{y}_{3} \mid \mathbf{x}_{2}\right) \propto \exp \left(\frac{-\left\|\mathbf{y}_{3}-\mathbf{H}_{4} \mathbf{x}_{2}-\mathbf{u}_{3,2}\right\|^{2}}{2 \sigma_{3,2}^{2}}\right), \\
& p\left(\mathbf{y}_{4} \mid \mathbf{x}_{2}\right) \propto \exp \left(\frac{-\left\|\mathbf{y}_{4}+\mathbf{H}_{3}\left(\mathbf{x}_{2}\right)^{*}-\mathbf{u}_{4,2}\right\|^{2}}{2 \sigma_{4,2}^{2}}\right) \\
& p\left(\mathbf{y}_{1} \mid \mathbf{x}_{3}\right) \propto \exp \left(\frac{-\left\|\mathbf{y}_{1}-\mathbf{H}_{3} \mathbf{x}_{3}-\mathbf{u}_{1,3}\right\|^{2}}{2 \sigma_{1,3}^{2}}\right), \\
& p\left(\mathbf{y}_{2} \mid \mathbf{x}_{3}\right) \propto \exp \left(\frac{-\left\|\mathbf{y}_{2}-\mathbf{H}_{4} \mathbf{x}_{3}-\mathbf{u}_{2,3}\right\|^{2}}{2 \sigma_{2,3}^{2}}\right), \\
& p\left(\mathbf{y}_{3} \mid \mathbf{x}_{3}\right) \propto \exp \left(\frac{-\left\|\mathbf{y}_{3}-\mathbf{H}_{1}\left(\mathbf{x}_{3}\right)^{*}-\mathbf{u}_{3,3}\right\|^{2}}{2 \sigma_{3,3}^{2}}\right), \\
& p\left(\mathbf{y}_{4} \mid \mathbf{x}_{3}\right) \propto \exp \left(\frac{-\left\|\mathbf{y}_{4}-\mathbf{H}_{2}\left(\mathbf{x}_{3}\right)^{*}-\mathbf{u}_{4,3}\right\|^{2}}{2 \sigma_{4,3}^{2}}\right) .
\end{aligned}
$$

According to (25)-(31), the process of the MP detector for OSTBC-SM with $R=3 / 4$ is detailed as follows.

Step 1: Initialize the probability $p_{t k}^{0}\left(s_{q}\right)$ by $p_{t k}^{0}\left(s_{q}\right)=$ $\frac{1}{Q}(\forall t, k, q)$, where $Q=2^{R_{p} T}$.

Step 2: Compute the messages $\mathbf{u}_{t, k}^{i},\left(\sigma_{t, k}^{2}\right)^{i}$ for $t=$ $(1,2,3,4), k=(1,2,3)$ via Appendix A using $p_{t k}^{i-1}\left(s_{q}\right)$.

Step 3: Compute the messages $p_{t k}^{i}\left(s_{q}\right)$ for $t=$ $(1,2,3,4), k=(1,2,3)$ using the values of $\mathbf{u}_{t, k}^{i},\left(\sigma_{t, k}^{2}\right)^{i}$ by

$$
p_{t k}^{i}\left(s_{q}\right)=\prod_{j=1, j \neq t}^{M} p\left(\mathbf{y}_{j} \mid \mathbf{x}_{k}=s_{q}\right) .
$$

Step 4: Repeat Steps 2 and 3 until $\left\|\mathbf{P}^{i}-\mathbf{P}^{i-1}\right\|^{2}<\varsigma$, where $\varsigma \in(0,1)$ is a small constant and $\mathbf{P}^{i}=\left[p_{t k}^{i}\left(s_{q}\right)\right]$ for $t=(1,2,3,4)$ and $k=(1,2,3)$.

Step 5: $\hat{q}=\underset{q \in(1, Q)}{\arg \max } \prod_{t=1}^{4} p\left(\mathbf{y}_{t} \mid \mathbf{x}_{k}=\mathbf{s}_{q}\right) ; \hat{\mathbf{x}}_{k}=\mathbf{s}_{\hat{q}}$.

2) Proposed MP detector for GSTBC-SM:

According to Fig. 2, (25) and the descriptions of MP detector for OSTBC-SM with $R=0.75$, the process of MP for GSTBC-SM is detailed in Algorithm 1, where $N_{\text {iter }}$ is the number of iterations and $\alpha \in(0,1)$ is a damping factor. According to Algorithm 1, for a specific GSTBC-SM structure, we can calculate the values of $\mathbf{u}_{t, k}^{i}$ and $\left(\sigma_{t, k}^{i}\right)^{2}$ by (25), which can further help to attain the conditional probabilities of line 10. Hence, the proposed MP detector is suitable for any GSTBC-SM systems. However, in [45], the authors indicate that MP detector is more suitable for the case of $K<T$. For the case of $K=T$, it may exhibit performance loss, which will be demonstrated in simulation results. In order to make the signal detection of the proposed GSTBC-SM system more comprehensive, a novel B-MMSE detector will be developed in the next subsection.

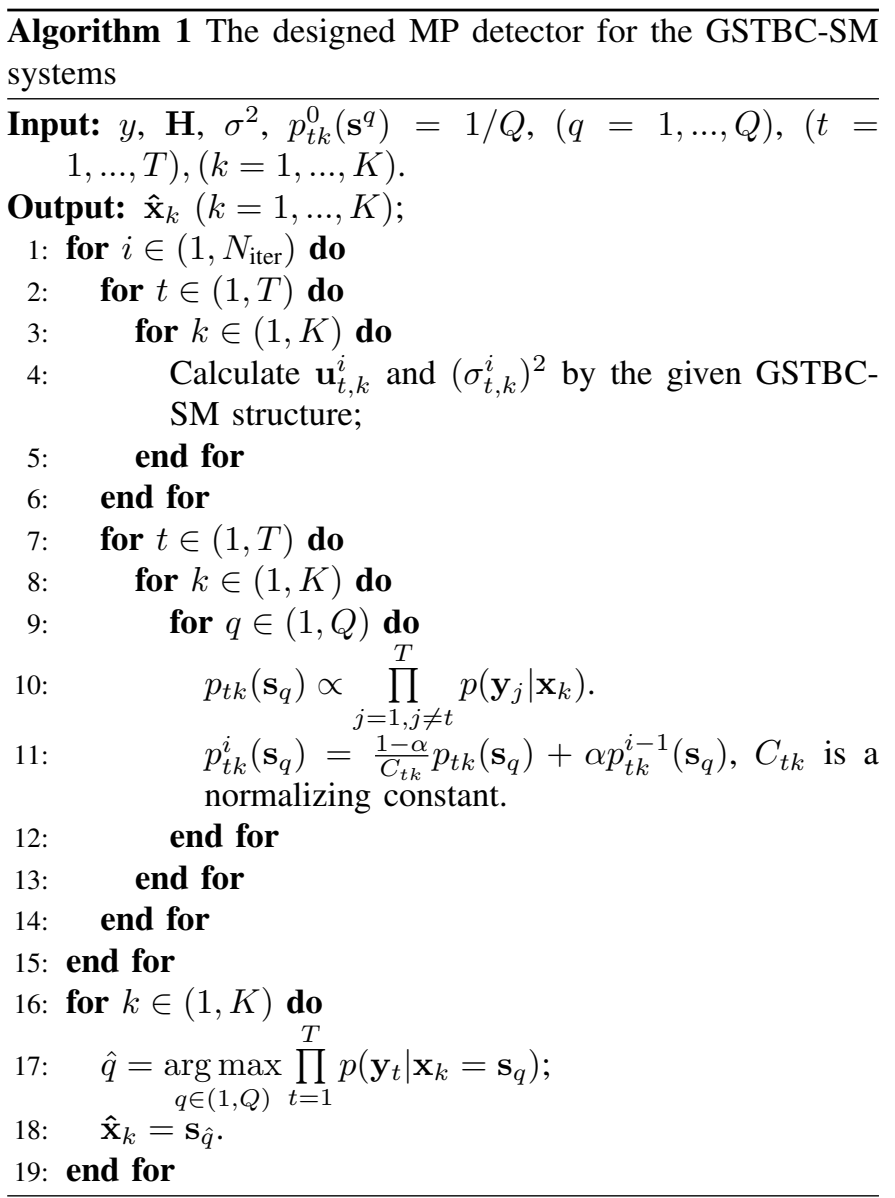

\section{B. Proposed B-MMSE detector}

In this section, low-complexity B-MMSE detector is designed for GSTBC-SM system. Firstly, the design for the QOSTBC-SM with $R=1$ is introduced, then its extension for the GSTBC-SM is developed. 
1) B-MMSE for Q-OSTBC-SM with $R=1$ :

In the Q-OSTBC-SM system, assuming that $\mathbf{Y}=\left[\mathbf{y}_{1}, \mathbf{y}_{2}, \mathbf{y}_{3}, \mathbf{y}_{4}\right], \quad \mathbf{H}=\left[\mathbf{H}_{1} \mathbf{H}_{2} \mathbf{H}_{3} \mathbf{H}_{4}\right]$, and $\mathbf{N}=\left[\mathbf{n}_{1}, \mathbf{n}_{2}, \mathbf{n}_{3}, \mathbf{n}_{4}\right]$, the Q-OSTBC-SM received signal can be formulated via (11) and (23) as

$$
\begin{aligned}
& \mathbf{y}_{1}=\mathbf{H}_{1} \mathbf{x}_{1}+\mathbf{H}_{2} \mathbf{x}_{2}+\mathbf{H}_{3} \mathbf{x}_{3} e^{j \phi}+\mathbf{H}_{4} \mathbf{x}_{4} e^{j \phi}+\mathbf{n}_{1}, \\
& \mathbf{y}_{2}=\mathbf{H}_{2} \mathbf{x}_{1}^{*}-\mathbf{H}_{1} \mathbf{x}_{2}^{*}-\mathbf{H}_{3} \mathbf{x}_{4}^{*} e^{-j \phi}+\mathbf{H}_{4} \mathbf{x}_{3}^{*} e^{-j \phi}+\mathbf{n}_{2}, \\
& \mathbf{y}_{3}=\mathbf{H}_{3} \mathbf{x}_{1}^{*}+\mathbf{H}_{4} \mathbf{x}_{2}^{*}-\mathbf{H}_{1} \mathbf{x}_{3}^{*} e^{-j \phi}-\mathbf{H}_{2} \mathbf{x}_{4}^{*} e^{-j \phi}+\mathbf{n}_{3}, \\
& \mathbf{y}_{4}=\mathbf{H}_{4} \mathbf{x}_{1}-\mathbf{H}_{3} \mathbf{x}_{2}-\mathbf{H}_{2} \mathbf{x}_{3} e^{j \phi}+\mathbf{H}_{1} \mathbf{x}_{4} e^{j \phi}+\mathbf{n}_{4} .
\end{aligned}
$$

To easy detection, (33) can be further represented as

$$
\underbrace{\left[\begin{array}{l}
\mathbf{y}_{1} \\
\mathbf{y}_{2}^{*} \\
\mathbf{y}_{3}^{*} \\
\mathbf{y}_{4}
\end{array}\right]}_{\hat{\mathbf{y}}}=\underbrace{\left[\begin{array}{cccc}
\mathbf{H}_{1} & \mathbf{H}_{2} & \mathbf{H}_{3} & \mathbf{H}_{4} \\
\mathbf{H}_{2}^{*}-\mathbf{H}_{1}^{*} & \mathbf{H}_{4}^{*} & -\mathbf{H}_{3}^{*} \\
\mathbf{H}_{3}^{*} & \mathbf{H}_{4}^{*} & -\mathbf{H}_{1}^{*}-\mathbf{H}_{2}^{*} \\
\mathbf{H}_{4}-\mathbf{H}_{3}-\mathbf{H}_{2} & \mathbf{H}_{1}
\end{array}\right]}_{\hat{\mathbf{H}}} \underbrace{\left[\begin{array}{c}
\mathbf{x}_{1} \\
\mathbf{x}_{2} \\
\mathbf{x}_{3} e^{j \phi} \\
\mathbf{x}_{4} e^{j \phi}
\end{array}\right]}_{\hat{\mathbf{x}}}+\underbrace{\left[\begin{array}{c}
\mathbf{n}_{1} \\
\mathbf{n}_{2}^{*} \\
\mathbf{n}_{3}^{*} \\
\mathbf{n}_{4}
\end{array}\right]}_{\hat{\mathbf{n}}} .
$$

It is obvious that there are lots of zeros in the vector $\hat{\mathbf{x}}$, so that (34) can be simplified as

$$
\hat{\mathbf{y}}=\hat{\mathbf{H}}_{\Omega} \mathbf{s}+\hat{\mathbf{n}}
$$

where $\Omega$ is the antenna combination and $\mathbf{s}$ is the symbol vector, which are expressed as

$$
\begin{aligned}
& \Omega=\left(q_{1}, n_{l}+q_{2}, 2 n_{l}+q_{3}, 3 n_{l}+q_{4}\right), \\
& \mathbf{s}=\left[s_{1} s_{2} s_{3} e^{j \phi} s_{4} e^{j \phi}\right]^{T},
\end{aligned}
$$

where $q_{l}$ denotes the activated index of $\mathbf{x}_{l}$ and $n_{l}=N_{t} / 4$. Then, the optimal antenna combination can be obtained as

$$
\hat{\Omega}=\arg \min _{\Omega \in \mathbb{I}}\left\|\hat{\mathbf{y}}-\hat{\mathbf{H}}_{\Omega} \mathbf{s}_{\Omega}\right\|^{2},
$$

where $\mathbb{I}$ is the set of antenna combination and $\mathbf{s}_{\Omega}$ is

$$
\mathbf{s}_{\Omega}=\left(\left[\hat{\mathbf{H}}_{\Omega}^{H} \hat{\mathbf{H}}_{\Omega}+\sigma^{2} \mathbf{I}_{4}\right]^{-1} \hat{\mathbf{H}}_{\Omega}^{H} \hat{\mathbf{y}}\right) .
$$

After obtaining the optimal antenna combination $\hat{\Omega}$, the symbol vector can be obtained as

$$
\hat{\mathbf{s}}=\left(\left[\hat{\mathbf{H}}_{\hat{\Omega}}^{H} \hat{\mathbf{H}}_{\hat{\Omega}}+\sigma^{2} \mathbf{I}_{4}\right]^{-1} \hat{\mathbf{H}}_{\hat{\Omega}}^{H} \hat{\mathbf{y}}\right) .
$$

To further reduce the complexity, if the condition satisfies

$$
\left\|\hat{\mathbf{y}}-\hat{\mathbf{H}}_{\Omega} \mathbf{s}_{\Omega}\right\|^{2} \leq V_{\mathrm{th}},
$$

where $V_{\text {th }}=\beta N_{r} \sigma^{2} T$, the detection process will terminate [44]. The parameter $\beta$ is a constant to balance the tradeoff between the computation complexity and performance.

2) B-MMSE for GSTBC-SM:

In the GSTBC-SM system, assuming that we have $\mathbf{Y}=\left[\mathbf{y}_{1}, \mathbf{y}_{2}, \cdots, \mathbf{y}_{T}\right], \mathbf{H}=\left[\mathbf{H}_{1} \mathbf{H}_{2} \cdots \mathbf{H}_{T}\right]$, and $\mathbf{N}=$

\begin{tabular}{|c|c|c|c|}
\hline \multicolumn{2}{|r|}{ Operations (25) } & Flops & Average times \\
\hline \multirow[t]{2}{*}{$\mathbf{u}_{t, k}^{i}$} & $\overline{\mathbf{x}}_{k}=\sum_{q=1}^{Q} s_{q} p_{t k}\left(s_{q}\right)$ & $2 Q$ & \multirow[t]{2}{*}{$(K-1) T K N_{\text {iter }}$} \\
\hline & $\mathbf{H}_{(k)} \overline{\mathbf{x}}_{k}$ & $6 N_{r}$ & \\
\hline \multirow[t]{2}{*}{$\left(\sigma_{t, k}^{2}\right)^{i}$} & $\overline{\mathbf{x}}_{k}^{2}=\sum_{q=1}^{Q}\left|s_{q}\right|^{2} p_{t k}\left(s_{q}\right)$ & $3 Q$ & \multirow[t]{2}{*}{$(K-1) T K N_{\text {iter }}$} \\
\hline & $\left\|\mathbf{H}_{(k)}\right\|^{2} \overline{\mathbf{x}}_{k}^{2}$ & $4 N_{r}$ & \\
\hline$p_{t, k}^{i}\left(s_{q}\right)$ & $p\left(\mathbf{y}_{j} \mid \mathbf{x}_{k}=s_{q}\right)$ & $14 N_{r}$ & $Q(T-1) K T N_{\text {iter }}$ \\
\hline Total & \multicolumn{3}{|c|}{$\left[\left(5 Q+10 N_{r}\right)(K-1)+14 N_{r} Q(T-1)\right] T K N_{\text {iter }}$} \\
\hline
\end{tabular}
$\left[\mathbf{n}_{1}, \mathbf{n}_{2}, \cdots, \mathbf{n}_{T}\right]$. If the received signal can be reformulated as

$$
\tilde{\mathbf{y}}=\tilde{\mathbf{H}} \tilde{\mathbf{x}}+\tilde{\mathbf{n}},
$$

where $\tilde{\mathbf{y}} \in \mathbb{C}^{N_{r} T \times 1}$ is associated with $\mathbf{y}_{1}, \mathbf{y}_{2}, \cdots, \mathbf{y}_{T}$, $\tilde{\mathbf{H}} \in \mathbb{C}^{N_{r} T \times n_{t} K}$ is associated with $\mathbf{H}_{1}, \mathbf{H}_{2}, \cdots, \mathbf{H}_{T}$ and $\tilde{\mathbf{n}} \in \mathbb{C}^{N_{r} T \times 1}$ is associated with $\mathbf{n}_{1}, \mathbf{n}_{2}, \cdots, \mathbf{n}_{T}$, then B-MMSE detector can be employed; Otherwise, MP detector is more attractive.
TABLE II

COMPLEXITY OF PROPOSED MP DETECTOR

\section{Complexity analysis}

In this subsection, the complexities of the proposed MP and B-MMSE detectors are evaluated in terms of real-valued Flops. For specific matrices $\mathbf{A} \in \mathbb{C}^{m \times n}, \mathbf{B} \in \mathbb{C}^{n \times p}, \mathbf{c} \in$ $\mathbb{C}^{n \times 1}$ and $\mathbf{d} \in \mathbb{C}^{n \times 1}$, the operations of $\mathbf{A B},\|\mathbf{c}\|_{F}^{2}$ and $\mathbf{c} \pm \mathbf{d}$ require $8 m n p-2 m p, 4 n-1$, and $2 n$ flops, respectively. The complexity of the ML detector can be expressed as

$$
C_{\mathrm{ML}}=\left(8 N_{r} K T+4 N_{r} T\right) 2^{R_{p} T} .
$$

According to (25) and Algorithm 1, the complexity of the proposed MP detector is expressed as

$$
C_{\mathrm{MP}}=\left[\left(5 Q+10 N_{r}\right)(K-1)+14 N_{r} Q(T-1)\right] T K N_{\mathrm{iter}},
$$

where $N_{\text {iter }}$ is the number of iterations and the detailed calculation process is presented in Table II.

On the other hand, the complexity of the B-MMSE detector is mainly imposed by the calculation of (39), whose complexity is $4 K^{3}+12 K^{2} N_{r}+7 K^{2}+6 N_{r} K$ [44]. Assuming the average number of MMSE detection of (39) is $N_{\text {avg }}$, the complexity of B-MMSE detector can be obtained as

$$
C_{\mathrm{B}-\mathrm{MMSE}}=N_{\mathrm{avg}}\left(4 K^{3}+12 K^{2} N_{r}+7 K^{2}+6 N_{r} K\right) .
$$

where $N_{\text {avg }}$ is the average number of the operation in (38).

\section{ABEP ANALYSIS FOR THE PROPOSED GSTBC-SM SYSTEMS}

In this section, ABEP upper bound is derived for our coherent GSTBC-SM system. Assuming that the transmitted and received signals of GSTBC-SM are $\mathbf{S}_{i}$ and $\mathbf{S}_{j}$, respectively, the ABEP upper bound is given by

$$
P_{b}=\frac{1}{T R_{p} 2^{T R_{p}}} \sum_{i=1}^{2^{T R_{p}}} \sum_{j=1, j \neq i}^{2^{T R_{p}}} d\left(\mathbf{S}_{i}, \mathbf{S}_{j}\right) P\left(\mathbf{S}_{i} \rightarrow \mathbf{S}_{j}\right),
$$

where $d\left(\mathbf{S}_{i}, \mathbf{S}_{j}\right)$ is the number of error bits and $P\left(\mathbf{S}_{i} \rightarrow \mathbf{S}_{j}\right)$ denotes the pairwise error probability (PEP). According to [36], the PEP can be obtained by

$$
\begin{aligned}
& P\left(\mathbf{S}_{i} \rightarrow \mathbf{S}_{j}\right)=\mathbf{E}\left(P\left(\mathbf{S}_{i} \rightarrow \mathbf{S}_{j}\right) \mid \mathbf{H}\right) \\
& =\mathbf{E}\left(Q\left(\sqrt{\frac{\|\mathbf{H} \Delta\|^{2}}{2 \sigma^{2}}}\right)\right) \\
& =\int_{\gamma} Q\left(\sqrt{\frac{\gamma}{2 \sigma^{2}}}\right) d_{\gamma},
\end{aligned}
$$


where $\mathbf{E}(\cdot)$ denotes expectation operator, $Q(x)=$ $\int_{x}^{\infty} \frac{1}{\sqrt{2 \pi}} e^{-x^{2} / 2} d_{x}, \gamma=\|\mathbf{H} \Delta\|^{2}$ with $\Delta=\left(\mathbf{S}_{i}-\mathbf{S}_{j}\right)$. According to [36], the value of $P\left(\mathbf{S}_{i} \rightarrow \mathbf{S}_{j}\right)$ is dominated by the MGF of $\gamma$ and the value of $Q(x)$, which will be introduced as follows.

\section{A. $M G F$ of $\gamma$}

The MGF of $\gamma$ is expressed as $M_{\gamma}(s)=\int_{\gamma} e^{\gamma s} f_{\gamma}(\gamma) d_{\gamma}$ and calculated as follows. First, the value of $\gamma$ is expressed as

$\gamma=\operatorname{Tr}\left[\mathbf{H} \Delta \Delta^{H} \mathbf{H}^{H}\right]=\operatorname{Tr}\left[\tilde{\mathbf{H}} \mathbf{V} \tilde{\mathbf{H}}^{H}\right]=\sum_{r=1}^{N_{r}} \sum_{n=1}^{N_{t}}\left|\lambda_{n} \tilde{\mathbf{H}}_{r n}\right|^{2}$,

where $\Delta \Delta^{H}=\mathbf{U V U}^{H}$ with $\mathbf{U U}^{H}=\mathbf{I}_{N_{t}}, \mathbf{V}=$ $\operatorname{diag}\left(\lambda_{1}, \cdots, \lambda_{N_{t}}\right), \tilde{\mathbf{H}}_{r n}$ denotes the $r$-th row and $n$-column of $\tilde{\mathbf{H}}=\mathbf{H U}$, and $\lambda_{n}$ denotes the $n$-th eigenvalues of $\Delta \Delta^{H}$. Assuming that $\gamma_{r}=\sum_{n=1}^{N_{t}}\left|\lambda_{n} \tilde{\mathbf{H}}_{r n}\right|^{2}$, we have $\gamma=\sum_{r=1}^{N_{r}} \gamma_{r}$, and the MGF of $\gamma_{r}$ is expressed as

$$
M_{\gamma}(s)=\int_{\gamma} e^{\gamma s} f_{\gamma}(\gamma) d_{\gamma}=\prod_{n=1}^{N_{r}} M_{\gamma_{r}}(s),
$$

where $M_{\gamma_{r}}(s)=\int_{\gamma_{r}} e^{\gamma_{r} s} f_{\gamma_{r}}\left(\gamma_{r}\right) d_{\gamma_{r}}$. Furthermore, the value of $\gamma_{r}$ can be reformulated as

$$
\gamma_{r}=\sum_{n=1}^{N_{t}}\left|\lambda_{n} \tilde{\mathbf{H}}_{r n}\right|^{2}=\sum_{n=1}^{\kappa} \lambda_{n}\left|\tilde{\mathbf{H}}_{r n}\right|^{2}=\sum_{n=1}^{\kappa} \Phi_{n} .
$$

where $\kappa$ is the number of nonzero eigenvalues and $\Phi_{n}=\lambda_{n}\left|\tilde{\mathbf{H}}_{r n}\right|^{2}$. Hence, the MGF of $\gamma_{r}$ can be obtained as

$$
M_{\gamma_{r}}(s)=\prod_{n=1}^{\kappa} M_{\Phi_{n}}(s) .
$$

Note that, we have

$$
2 \Phi_{n} / \lambda_{n}=2\left|\tilde{\mathbf{H}}_{r n}\right|^{2} \in \chi^{2}(2) \rightarrow M_{\chi}(s)=(1-2 s)^{-1} .
$$

Then, the MGF of $\Phi_{n}$ is expressed as $M_{\Phi_{n}}(s)=\left(1-\lambda_{n} s\right)^{-1}$. As a result, the MGF of $\gamma$ can be obtained as

$$
M_{\gamma}(s)=\prod_{r=1}^{N_{r}} \prod_{n=1}^{\kappa} M_{\Phi_{n}}(s)=\prod_{n=1}^{\kappa}\left(1-\lambda_{n} s\right)^{-N_{r}},
$$

where $\lambda_{1}, \ldots, \lambda_{\kappa}$ are the nonzero eigenvalues.

\section{B. Accurate upper bound of $A B E P$}

According to [36], $Q(x)$ can be accurately represented as

$$
Q(x)=\frac{1}{\pi} \int_{0}^{\frac{\pi}{2}} \exp \left(-\frac{x^{2}}{2 \sin \theta^{2}}\right) d_{\theta} .
$$

Then, the PEP value can be obtained as

$$
\begin{aligned}
& P\left(\mathbf{S}_{i} \rightarrow \mathbf{S}_{i}\right)=\frac{1}{\pi} \int_{0}^{\pi / 2} M_{\gamma}\left(\frac{-1}{4 \sigma^{2} \sin ^{2} \theta}\right) d_{\theta} \\
& =\frac{1}{\pi} \int_{0}^{\pi / 2} \prod_{n=1}^{\kappa}\left(1+\frac{\lambda_{n}}{4 \sigma^{2} \sin ^{2} \theta}\right)^{-N_{r}} d_{\theta} .
\end{aligned}
$$

By substituting (54) into (45), the accurate ABEP upper bound can be obtained.

\section{Approximate upper bound of $A B E P$}

Since it is difficult to obtain the closed form of (54), we need to calculate the integration in the calculation of the upper bound, which imposes extra complexity. In order to further simplify the calculation of the ABEP upper bound, the value of $Q(x)$ can be approximated as [46]

$$
Q(x) \approx \frac{1}{12} e^{-\frac{x^{2}}{2}}+\frac{1}{4} e^{-\frac{2 x^{2}}{3}} .
$$

Then, the value of $P\left(\mathbf{x}_{i}^{t} \rightarrow \mathbf{x}_{i}^{j}\right)$ can be derived as

$$
\begin{aligned}
& P\left(\mathbf{x}_{i}^{t} \rightarrow \mathbf{x}_{i}^{j}\right) \\
& \approx \int_{\gamma}\left(\frac{1}{12} e^{-\frac{\gamma}{4 \sigma^{2}}}+\frac{1}{4} e^{-\frac{\gamma}{3 \sigma^{2}}}\right) f_{\gamma}(\gamma) d \gamma \\
& =\frac{1}{12} M_{\gamma}\left(-\frac{1}{4 \sigma^{2}}\right)+\frac{1}{4} M_{\gamma}\left(-\frac{1}{3 \sigma^{2}}\right), \\
& =\frac{1}{12} \prod_{n=1}^{\kappa}\left(1+\frac{\lambda_{n}}{4 \sigma^{2}}\right)^{-N_{r}}+\frac{1}{4} \prod_{n=1}^{\kappa}\left(1+\frac{\lambda_{n}}{3 \sigma^{2}}\right)^{-N_{r}} .
\end{aligned}
$$

By substituting (56) into (45), the approximate ABEP upper bound can be obtained.

\section{Simulation Results}

In this section, we conduct simulations to verify the performances of our proposed GSTBC-SM systems in the context of different antenna configurations, where the perfect channel state information is assumed at the receiver.

\section{A. Performance comparison of small-scale MIMO configura- tions}

In this subsection, we compare the performances of the proposed GSTBC-SM systems to those of the conventional GSTBC and space shift keying (SSK) systems [47] in the context of small-scale MIMO configurations. Moreover, both the accurate and approximate ABEP upper bound are added for benchmarkers. It can be observed from Figs. 3 and 6 that, the derived approximate upper bound approaches the accurate upper bound and both are closely tight with simulation results upon increasing the SNR values.

Specifically, Fig. 3 and 4 compare the performances of the proposed GSTBC-SM systems to those of conventional GSTBC and SSK systems at the throughput of $3 \mathrm{bpcu}$ in conjunction with $N_{r}=1$ and $N_{r}=2$, respectively. To achieve 3 bpcu, $\left(N_{t}, K, M, T\right)=(8,4,4,4)$, $(8,3,8,4)$ and $(8,2,2,2)$ are employed for proposed QOSTBC-SM, OSTBC-SM, Alamouti-SM [36] systems, while $\left(N_{t}, K, M, T\right)=(4,4,8,4),(4,3,16,4)$ and $(2,2,8,2)$ are used for conventional Q-OSTBC, OSTBC and Alamouti systems. It can be observed from Figs. 3-4 that, Q-OSTBC-SM with $\pi / 4$ performs the best and outperforms corresponding Q-OSTBC by $8 \mathrm{~dB}$ and $5 \mathrm{~dB}$ at $\mathrm{BER}=10^{-5}$ for the case of $N_{r}=1$ and $N_{r}=2$, respectively. OSTBC-SM outperforms corresponding OSTBC by around $6 \mathrm{~dB}$ at $\mathrm{BER}=10^{-5}$ for both the cases of $N_{r}=1$ and $N_{r}=2$. Since the diversity order of proposed GSTBC-SM is four, which is larger than that of Alamouti-SM, Alamouti and SSK schemes, all the proposed GSTBC-SM systems are capable of providing significant performance gains over the Alamouti-SM, Alamouti and SSK systems. 


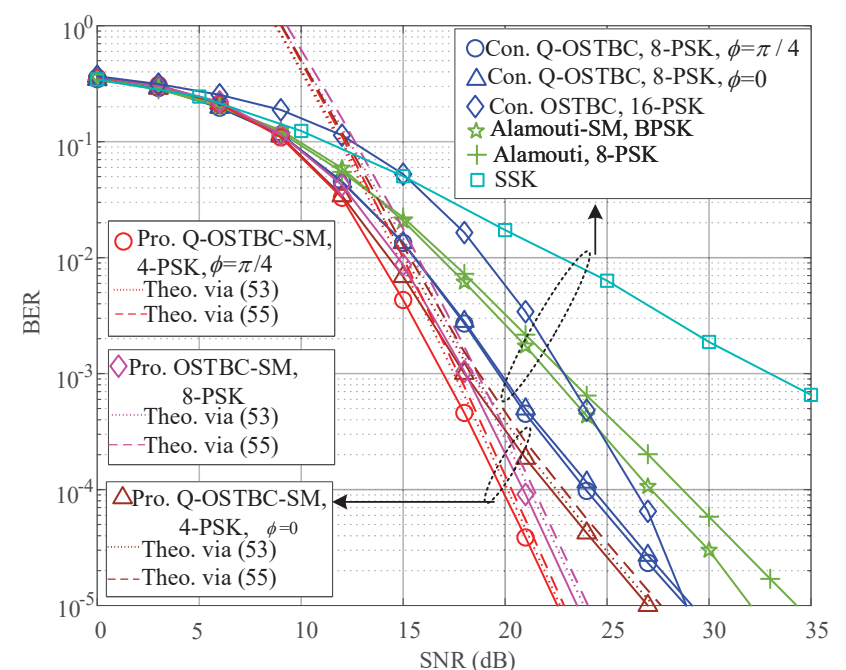

Fig. 3. Performance comparisons for proposed GSTBC-SM systems and conventional GSTBC systems having $N_{t}=8 N_{r}=1$ at $3 \mathrm{bpcu}$.

Figs. 5-6 compare the performances of the proposed GSTBC-SM systems to those of conventional GSTBC and SSK systems at the throughput of $4 \mathrm{bpcu}$. To achieve 4 bpcu, $\left(N_{t}, K, M, T\right)=(16,4,4,4)$, and $(16,2,2,2)$ are employed for Q-OSTBC-SM, Alamouti-SM [36], while $\left(N_{t}, K, M, T\right)=(4,4,16,4)$, and $(2,2,16,2)$ are employed for Q-OSTBC and Alamouti. Since it is difficult for OSTBC$\mathrm{SM}$ and OSTBC systems to attain $4 \mathrm{bpcu}$, the performance of them are not included for comparison. It can be observed from Fig. 4 that, Q-OSTBC-SM with $\phi=\pi / 4$ still performs the best and outperforms corresponding Q-OSTBC by around $8 \mathrm{~dB}$ at BER $=10^{-5}$ for both the cases of $N_{r}=1$ and $N_{r}=2$. As $N_{r}$ increases, the performance of Q-OSTBC-SM with $\phi=0$ approaches that of Q-OSTBC-SM with $\phi=\pi / 4$. It is also obvious that the proposed Q-OSTBC-SM system with $\phi=\pi / 4$ outperforms Alamouti-SM, Alamouti and SSK systems by $5 \mathrm{~dB}, 12 \mathrm{~dB}$ and $13 \mathrm{~dB}$ at $\mathrm{BER}=10^{-5}$ for the case of $N_{r}=2$, respectively. The performance advantages become more dominant for the case of $N_{r}=1$.

Fig. 7 compares the performances of the proposed QOSTBC-SM systems to those of SSK-vertical bell labs spacetime (SSK-VBLAST) [47] systems having $N_{t}=8$ at the throughput of $4 \mathrm{bpcu}$. To achieve $4 \mathrm{bpcu},\left(N_{t}, K, M, T\right)=$ $(8,4,8,4)$ and $(8,4,1,1)$ are employed for Q-OSTBC-SM and SSK-VBLAST systems, respectively. It is obvious that the proposed Q-OSTBC-SM system with $\phi=\pi / 4$ outperforms SSK-VBLAST systems by $10 \mathrm{~dB}$ and $20 \mathrm{~dB}$ at $\mathrm{BER}=10^{-4}$ for the case of $N_{r}=2$ and $N_{r}=1$, respectively.

\section{B. Performance and complexity comparisons between different detectors of proposed GSTBC-SM systems}

In order to achieve near optimal performance with significantly reduced complexity in the context of massive MIMO communication, low-complexity MP and B-MMSE detectors are designed for our OSTBC-SM and Q-OSTBC-SM sys-

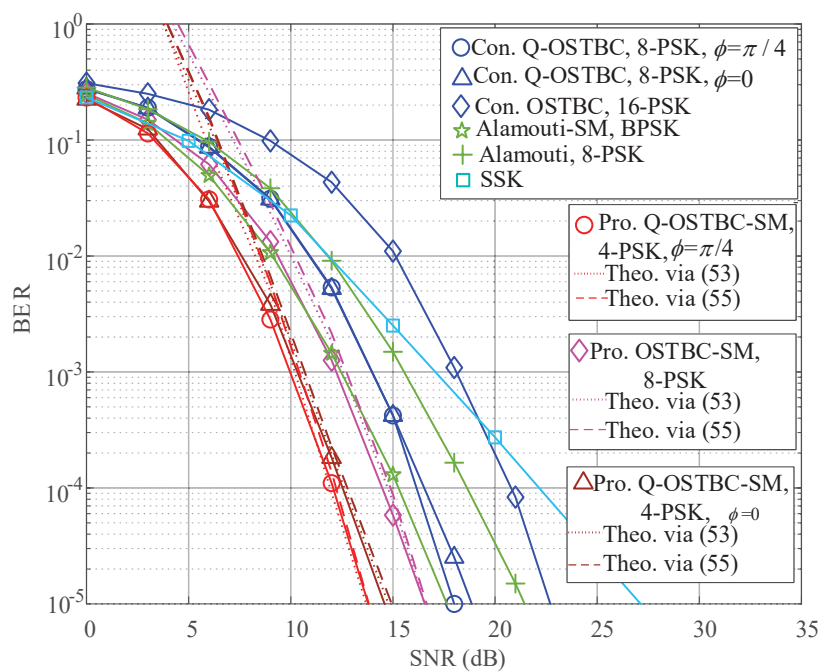

Fig. 4. Performance comparisons for proposed GSTBC-SM systems and conventional GSTBC systems having $N_{t}=8 N_{r}=2$ at $3 \mathrm{bpcu}$.

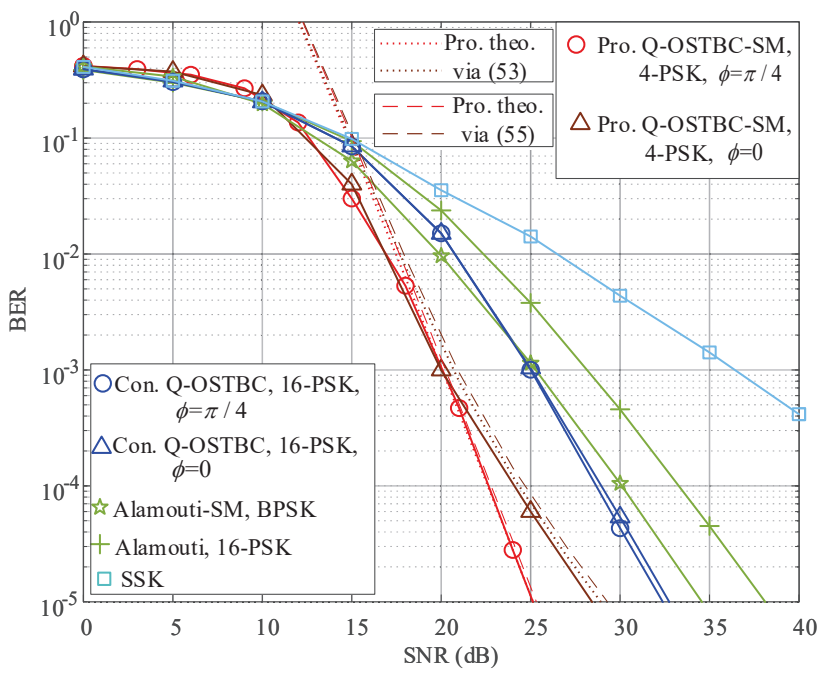

Fig. 5. Performance comparisons for proposed GSTBC-SM systems and conventional GSTBC and SSK systems having $N_{t}=16 N_{r}=1$ at $4 \mathrm{bpcu}$.

tems, respectively. In this subsection, the performance and complexity of the proposed low-complexity detectors are compared with the ML detector in Figs. 8-9 and Table III, respectively, where we have $C_{r}=\left(C_{\mathrm{ML}}-C_{\mathrm{MP}}\right) / C_{\mathrm{ML}}$ and $C_{r}=\left(C_{\mathrm{ML}}-C_{\mathrm{B}-\mathrm{MMSE}}\right) / C_{\mathrm{ML}}$ for the proposed OSTBC-SM and Q-OSTBC-SM, respectively.

Specifically, Fig. 8 compares the performances of the proposed MP and ML detectors for the QPSK-aided OSTBCSM systems with $N_{t}=8$ and $N_{t}=16$. It can be observed from Fig. 8 that, the performance of the proposed MP detector approaches that of the ML detector for the cases of $N_{t}=8, N_{r}=4$ and $N_{t}=16, N_{r}=6$. For the case of small number of receiver antennas, the proposed MP detector suffer from near $2 \mathrm{~dB}$ performance loss over the ML detector. It can 


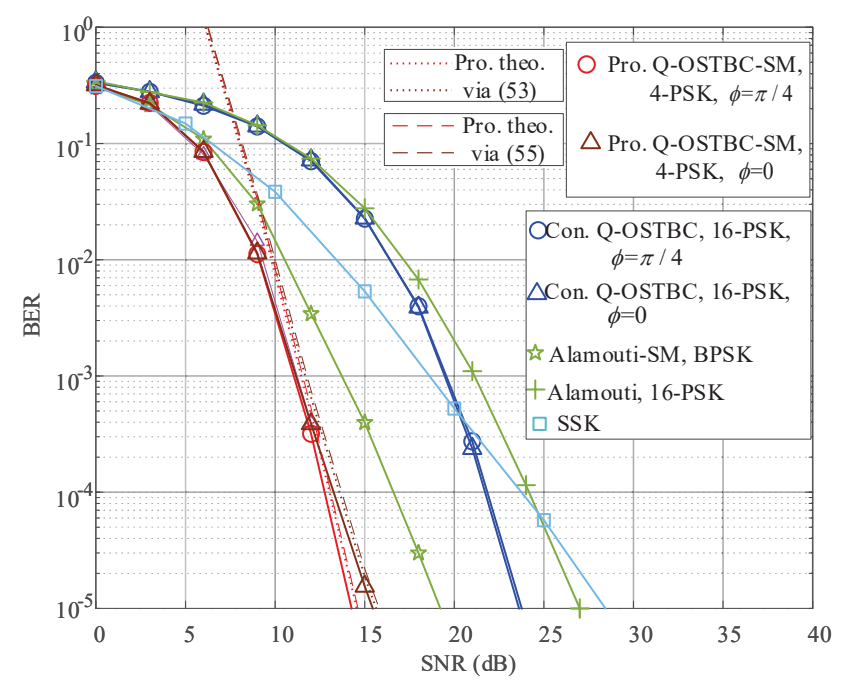

Fig. 6. Performance comparisons for proposed GSTBC-SM systems and conventional GSTBC and SSK systems having $N_{t}=16 N_{r}=2$ at 4 bpcu.

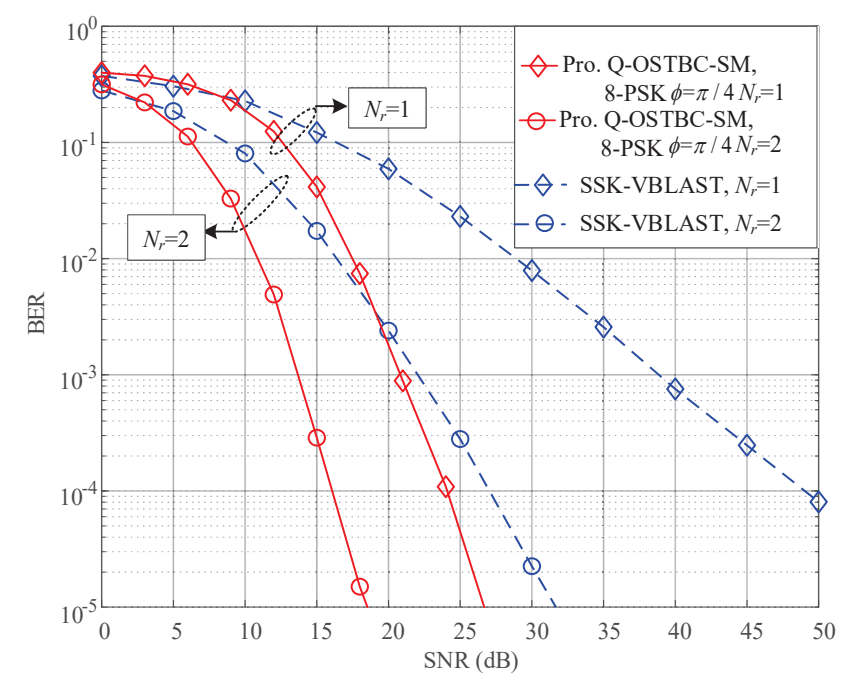

Fig. 7. Performance comparisons for proposed GSTBC-SM systems and SSK-VBLAST systems having $N_{t}=8$ at 4 bpcu.

be observed from Table III that, the proposed MP detector reduces the complexity to about $31.7 \% \sim 7.7 \%$ of that of the ML detector.

Fig. 9 presents the performances of the proposed MP, the proposed B-MMSE and ML detectors for the QPSK-aided QOSTBC-SM systems with $N_{t}=8$ at 3 bpcu. It can be observed from Fig. 9 that, the performance of the proposed B-MMSE detector depends on the value of $\beta$. To balance a tradeoff between the performance and the complexity, it is better to set $\beta=1.5, \beta=1.2$ and $\beta=0.9$ for the cases of $N_{r}=2, N_{r}=4$ and $N_{r}=8$, respectively. It can be observed from Fig. 9 that, the performance of the B-MMSE detector with the appropriate value of $\beta$ always approaches that of the ML detector and outperforms that of the proposed MP detector. This is because
TABLE III

COMPLEXITY COMPARISON OF THE PROPOSED DETECTORS WITH ML DETECTOR IN TERMS OF FLOPS.

\begin{tabular}{|c|c|c|c|c|c|}
\hline Scheme & $\begin{array}{c}\text { Antenna } \\
\text { setup }\end{array}$ & ML & MP & B-MMSE & $C_{r}$ \\
\hline \multirow[t]{2}{*}{$\begin{array}{c}\text { OSTBC } \\
\text {-SM }\end{array}$} & $\begin{array}{l}N_{t}=8 \\
N_{r}=4\end{array}$ & $2.3 \times 10^{5}$ & $7.2 \times 10^{4}$ & - & $68.53 \%$ \\
\hline & $\begin{array}{c}N_{t}=16 \\
N_{r}=4\end{array}$ & $1.8 \times 10^{6}$ & $1.4 \times 10^{5}$ & - & $92.34 \%$ \\
\hline \multirow[t]{2}{*}{$\begin{array}{c}\text { Q-OSTBC } \\
\text {-SM }\end{array}$} & $\begin{array}{l}N_{t}=8 \\
N_{r}=2\end{array}$ & $1.2 \times 10^{6}$ & - & $\begin{array}{c}7200 \\
\beta=1.5\end{array}$ & $99.39 \%$ \\
\hline & $\begin{array}{l}N_{t}=8 \\
N_{r}=4\end{array}$ & $2.4 \times 10^{6}$ & - & $\begin{array}{c}13552 \\
\beta=1.2\end{array}$ & $99.43 \%$ \\
\hline
\end{tabular}
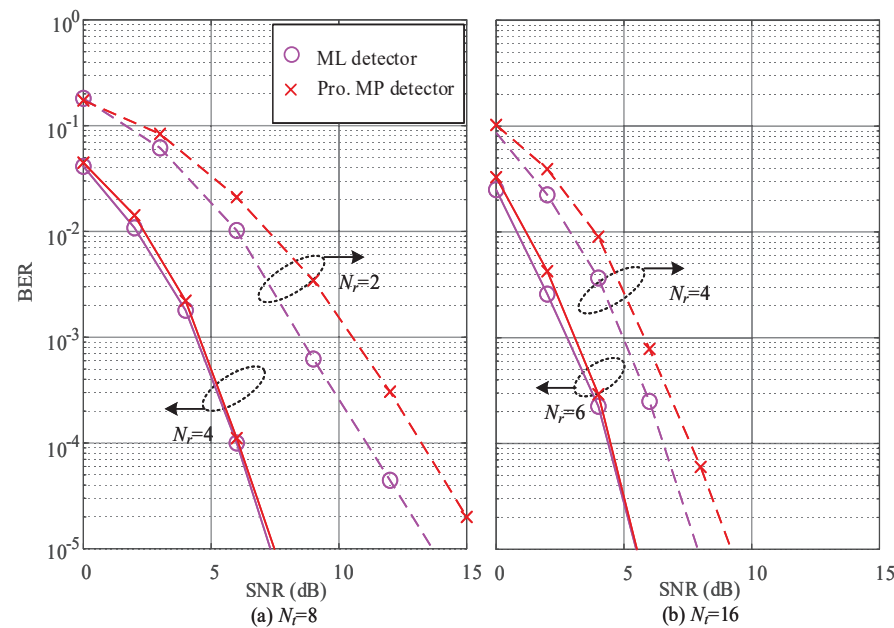

Fig. 8. Performance comparisons for OSTBC-SM systems associated with different detectors.

that the performance of the MP detector is associated with the value of $N_{r}$ and $K$. By further increasing the value of $N_{r}$ or decreasing the value of $K$, its performance is able to approach ML detector's counterpart. It can be observed from Table III that, the proposed B-MMSE detector reduces the complexity to about $0.5 \% \sim 0.6 \%$ of that of the ML detector.

In a short, the B-MMSE detector is only suitable for the GSTBC-SM systems whose received signal can be expressed by (41), while MP is suitable for any GSTBC-SM system. For a specific GSTBC-SM system, if B-MMSE detector can be employed, B-MMSE is the more attractive than MP detector; Otherwise, the proposed MP detector is more suitable.

\section{Performance comparison of massive MIMO configurations}

In this subsection, the performance comparisons in massive MIMO antenna configurations are presented, where the ML detector becomes impractical. Therefore, only the performances of the low-complexity detectors are included for comparison. For fair comparison, both OSTBC-SM and OSTBC systems employ our proposed MP detectors, while both QOSTBC-SM and Q-OSTBC systems employ our proposed B-MMSE detectors. Fig. 10 compares the performances of the proposed OSTBC-SM systems to those of conventional OSTBC systems at the throughput of $3.75 \mathrm{bpcu}$ and 4.5 bpcu, respectively. $\left(N_{t}, K, M, N_{r}, T\right)=(32,3,4,6,4)$, and $(64,3,4,6,4)$ are employed for OSTBC-SM, while 


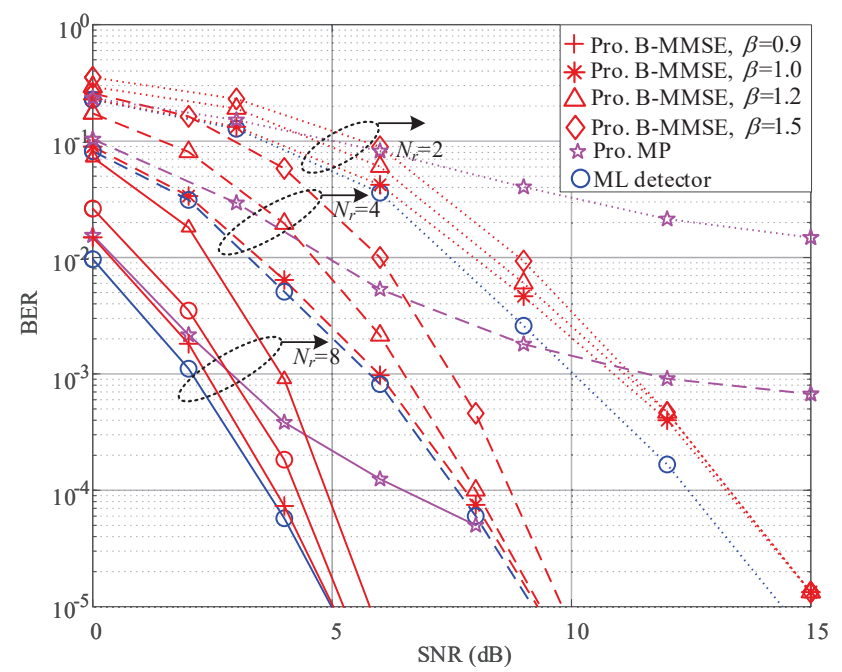

Fig. 9. Performance comparisons for Q-OSTBC-SM systems associated with different detectors at $3 \mathrm{bpcu}$.

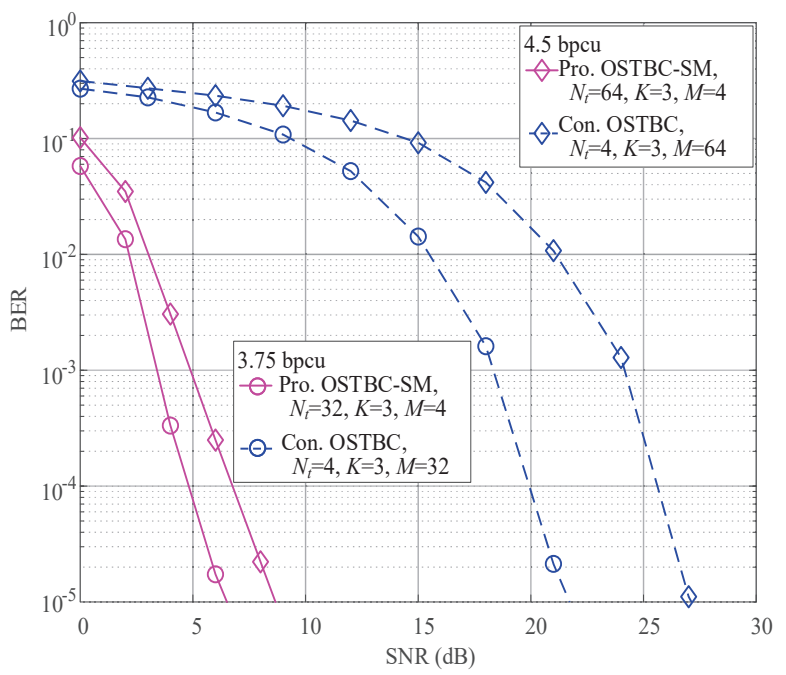

Fig. 10. Performance comparisons for proposed OSTBC-SM systems and conventional OSTBC systems at 3.75 bpcu and 4.5 bpcu.

$\left(N_{t}, K, M, N_{r}, T\right)=(4,3,32,6,4)$, and $(4,3,64,6,4)$ are used for conventional OSTBC. For the case of 3 RF chains, it can be observed from Fig. 10 that the proposed OSTBCSM system outperforms the conventional OSTBC system by around $15 \mathrm{~dB}$ and $20 \mathrm{~dB}$ at $3.75 \mathrm{bpcu}$ and $4.5 \mathrm{bpcu}$, respectively, at $\mathrm{BER}=10^{-5}$.

Fig. 11 compares the performance of the proposed QOSTBC-SM system to that of conventional Q-OSTBC, SSK and $\mathrm{ZF}$ precoded MIMO systems at the throughput of 6 bpcu. $\left(N_{t}, K, M, N_{r}, T\right)=(64,4,4,2,4)$ is employed for Q-OSTBC-SM, $\left(N_{t}, K, M, N_{r}, T\right)=(4,4,64,2,4)$ is used for conventional Q-OSTBC systems, and $\left(N_{t}, M, N_{r}, T\right)=$ $(64,8,2,1)$ is employed for ZF precoded MIMO. It can be observed from Fig. 11 that the proposed Q-OSTBC-SM system outperforms the conventional ZF precoded MIMO [48], SSK

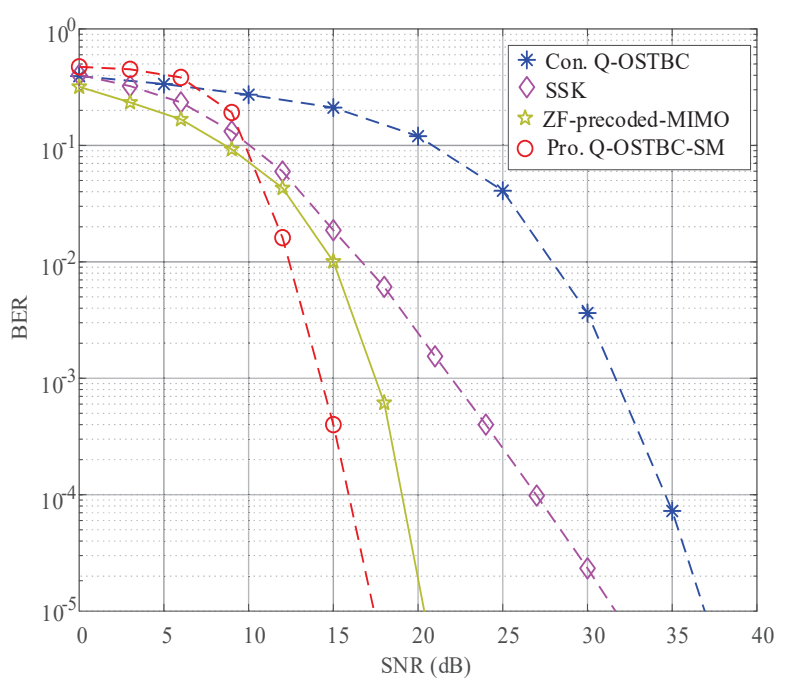

Fig. 11. Performance comparisons for proposed Q-OSTBC-SM system and conventional Q-OSTBC, SSK and ZF precoded MIMO systems at 6 bpcu.

and Q-OSTBC systems by around $3 \mathrm{~dB}, 13 \mathrm{~dB}$ and $20 \mathrm{~dB}$ at $\mathrm{BER}=10^{-5}$.

\section{CONCLUSIONS}

In this paper, we proposed a novel GSTBC-SM scheme for open-loop massive MIMO downlink communication systems, which is capable of attaining high throughput and diversity gain even with small number of RF chains. Then, the performances of the specific OSTBC-SM and Q-OSTBC-SM systems were investigated, while low-complexity detectors were designed in the context of massive MIMO antenna configurations. Both simulation and theoretical results show that the proposed GSTBC-SM scheme provides considerable performance gains over the corresponding STBC schemes. Moreover, the proposed GSTBC-SM scheme employing a large number of TAs provides $20 \mathrm{~dB}$ gain over the GSTBC system with identical number of RF chains and throughput.

\section{APPENDIX A}

The CALCULATION OF $u_{t, k}^{i}$ AND $\sigma_{t, k}^{i}$ IN (25)

In this Appendix, the values of $u_{t, k}^{i}$ and $\sigma_{t, k}^{i}$ in MP detector for OSTBC-SM system are calculated by (26)-(28) as follows

$$
\begin{aligned}
& u_{1,1}^{i}=\sum_{q=1}^{Q} \mathbf{H}_{2} \mathbf{s}_{q} p_{12}^{i-1}\left(\mathbf{s}_{q}\right)+\sum_{q=1}^{Q} \mathbf{H}_{3} \mathbf{s}_{q} p_{13}^{i-1}\left(\mathbf{s}_{q}\right), \\
& \sigma_{1,1}^{i}=\sum_{q=1}^{Q}\left\|\mathbf{H}_{2} \mathbf{s}_{q}\right\|^{2} p_{12}^{i-1}\left(\mathbf{s}_{q}\right)-\left\|\sum_{q=1}^{Q} \mathbf{H}_{2} \mathbf{s}_{q} p_{12}^{i-1}\left(\mathbf{s}_{q}\right)\right\|^{2} \\
& +\sum_{q=1}^{Q}\left\|\mathbf{H}_{3} \mathbf{s}_{q}\right\|^{2} p_{13}^{i-1}\left(\mathbf{s}_{q}\right)-\left\|\sum_{q=1}^{Q} \mathbf{H}_{3} \mathbf{s}_{q} p_{13}^{i-1}\left(\mathbf{s}_{q}\right)\right\|^{2}+\sigma^{2},
\end{aligned}
$$




$$
\begin{aligned}
& u_{2,1}^{i}=-\sum_{q=1}^{Q} \mathbf{H}_{1} \mathbf{s}_{q}^{*} p_{22}^{i-1}\left(\mathbf{s}_{q}\right)+\sum_{q=1}^{Q} \mathbf{H}_{4} \mathbf{s}_{q} p_{23}^{i-1}\left(\mathbf{s}_{q}\right), \\
& \sigma_{2,1}^{i}=\sum_{q=1}^{Q}\left\|\mathbf{H}_{1} \mathbf{s}_{q}^{*}\right\|^{2} p_{22}^{i-1}\left(\mathbf{s}_{q}\right)-\left\|\sum_{q=1}^{Q} \mathbf{H}_{1} \mathbf{s}_{q}^{*} p_{22}^{i-1}\left(\mathbf{s}_{q}\right)\right\|^{2} \\
& +\sum_{q=1}^{Q}\left\|\mathbf{H}_{4} \mathbf{s}_{q}\right\|^{2} p_{23}^{i-1}\left(\mathbf{s}_{q}\right)-\left\|\sum_{q=1}^{Q} \mathbf{H}_{4} \mathbf{s}_{q} p_{23}^{i-1}\left(\mathbf{s}_{q}\right)\right\|^{2}+\sigma^{2}, \\
& u_{3,1}^{i}=\sum_{q=1}^{Q} \mathbf{H}_{4} \mathbf{s}_{q} p_{32}^{i-1}\left(\mathbf{s}_{q}\right)+\sum_{q=1}^{Q} \mathbf{H}_{1} \mathbf{s}_{q}^{*} p_{33}^{i-1}\left(\mathbf{s}_{q}\right), \\
& \sigma_{3,1}^{i}=\sum_{q=1}^{Q}\left\|\mathbf{H}_{4} \mathbf{s}_{q}\right\|^{2} p_{32}^{i-1}\left(\mathbf{s}_{q}\right)-\left\|\sum_{q=1}^{Q} \mathbf{H}_{4} \mathbf{s}_{q} p_{32}^{i-1}\left(\mathbf{s}_{q}\right)\right\|^{2} \\
& +\sum_{q=1}^{Q}\left\|\mathbf{H}_{1} \mathbf{s}_{q}^{*}\right\|^{2} p_{33}^{i-1}\left(\mathbf{s}_{q}\right)-\left\|\sum_{q=1}^{Q} \mathbf{H}_{1} \mathbf{s}_{q}^{*} p_{33}^{i-1}\left(\mathbf{s}_{q}\right)\right\|^{2} \\
& +\sigma^{2},
\end{aligned}
$$$$
\begin{aligned}
& u_{4,1}^{i}=\sum_{q=1}^{Q} \mathbf{H}_{2} \mathbf{s}_{q}^{*} p_{43}^{i-1}\left(\mathbf{s}_{q}\right)-\sum_{q=1}^{Q} \mathbf{H}_{3} \mathbf{s}_{q}^{*} p_{42}^{i-1}\left(\mathbf{s}_{q}\right), \\
& \sigma_{4,1}^{i}=\sum_{q=1}^{Q}\left\|\mathbf{H}_{2} \mathbf{s}_{q}^{*}\right\|^{2} p_{43}^{i-1}\left(\mathbf{s}_{q}\right)-\left\|\sum_{q=1}^{Q} \mathbf{H}_{2} \mathbf{s}_{q}^{*} p_{43}^{i-1}\left(\mathbf{s}_{q}\right)\right\|^{2} \\
& +\sum_{q=1}^{Q}\left\|\mathbf{H}_{3} \mathbf{s}_{q}^{*}\right\|^{2} p_{42}^{i-1}\left(\mathbf{s}_{q}\right)-\left\|\sum_{q=1}^{Q} \mathbf{H}_{3} \mathbf{s}_{q}^{*} p_{42}^{i-1}\left(\mathbf{s}_{q}\right)\right\|^{2}+\sigma^{2},
\end{aligned}
$$$$
u_{1,2}^{i}=\sum_{q=1}^{Q} \mathbf{H}_{1} \mathbf{s}_{q} p_{11}^{i-1}\left(\mathbf{s}_{q}\right)+\sum_{q=1}^{Q} \mathbf{H}_{3} \mathbf{s}_{q} p_{13}^{i-1}\left(\mathbf{s}_{q}\right),
$$$$
\sigma_{1,2}^{i}=\sum_{q=1}^{Q}\left\|\mathbf{H}_{1} \mathbf{s}_{q}\right\|^{2} p_{11}^{i-1}\left(\mathbf{s}_{q}\right)-\left\|\sum_{q=1}^{Q} \mathbf{H}_{1} \mathbf{s}_{q} p_{13}^{i-1}\left(\mathbf{s}_{q}\right)\right\|^{2}
$$$$
+\sum_{q=1}^{Q}\left\|\mathbf{H}_{3} \mathbf{s}_{q}\right\|^{2} p\left(\mathbf{x}_{3}^{q}\right)-\left\|\sum_{q=1}^{Q} \mathbf{H}_{3} \mathbf{s}_{q} p_{13}^{i-1}\left(\mathbf{s}_{q}\right)\right\|^{2}+\sigma^{2},
$$$$
u_{2,2}^{i}=\sum_{q=1}^{Q} \mathbf{H}_{2} \mathbf{s}_{q}^{*} p_{21}^{i-1}\left(\mathbf{s}_{q}\right)+\sum_{q=1}^{Q} \mathbf{H}_{4} \mathbf{s}_{q} p_{23}^{i-1}\left(\mathbf{s}_{q}\right),
$$$$
\sigma_{2,2}^{i}=\sum_{q=1}^{Q}\left\|\mathbf{H}_{2} \mathbf{s}_{q}^{*}\right\|^{2} p_{21}^{i-1}\left(\mathbf{s}_{q}\right)-\left\|\sum_{q=1}^{Q} \mathbf{H}_{2} \mathbf{s}_{q}^{*} p_{21}^{i-1}\left(\mathbf{s}_{q}\right)\right\|^{2}
$$$$
+\sum_{q=1}^{Q}\left\|\mathbf{H}_{4} \mathbf{s}_{q}\right\|^{2} p_{23}^{i-1}\left(\mathbf{s}_{q}\right)-\left\|\sum_{q=1}^{Q} \mathbf{H}_{4} \mathbf{s}_{q} p_{23}^{i-1}\left(\mathbf{s}_{q}\right)\right\|^{2}+\sigma^{2},
$$$$
u_{3,2}^{i}=-\sum_{q=1}^{Q} \mathbf{H}_{3} \mathbf{s}_{q}^{*} p_{31}^{i-1}\left(\mathbf{s}_{q}\right)+\sum_{q=1}^{Q} \mathbf{H}_{1} \mathbf{s}_{q}^{*} p_{33}^{i-1}\left(\mathbf{s}_{q}\right),
$$$$
\sigma_{3,2}^{i}=\sum_{q=1}^{Q}\left\|\mathbf{H}_{3} \mathbf{s}_{q}^{*}\right\|^{2} p_{31}^{i-1}\left(\mathbf{s}_{q}\right)-\left\|\sum_{q=1}^{Q} \mathbf{H}_{3} \mathbf{s}_{q}^{*} p_{31}^{i-1}\left(\mathbf{s}_{q}\right)\right\|^{2}
$$$$
+\sum_{q=1}^{Q}\left\|\mathbf{H}_{1} \mathbf{s}_{q}^{*}\right\|^{2} p_{33}^{i-1}\left(\mathbf{s}_{q}\right)-\left\|\sum_{q=1}^{Q} \mathbf{H}_{1} \mathbf{s}_{q}^{*} p_{33}^{i-1}\left(\mathbf{s}_{q}\right)\right\|^{2}+\sigma^{2},
$$$$
u_{4,2}^{i}=\sum_{q=1}^{Q} \mathbf{H}_{4} \mathbf{s}_{q} p_{41}^{i-1}\left(\mathbf{s}_{q}\right)+\sum_{q=1}^{Q} \mathbf{H}_{2} \mathbf{s}_{q}^{*} p_{43}^{i-1}\left(\mathbf{s}_{q}\right),
$$$$
\sigma_{4,2}^{i}=\sum_{q=1}^{Q}\left\|\mathbf{H}_{4} \mathbf{s}_{q}\right\|^{2} p_{41}^{i-1}\left(\mathbf{s}_{q}\right)-\left\|\sum_{q=1}^{Q} \mathbf{H}_{4} \mathbf{s}_{q} p_{41}^{i-1}\left(\mathbf{s}_{q}\right)\right\|^{2}
$$$$
+\sum_{q=1}^{Q}\left\|\mathbf{H}_{2} \mathbf{s}_{q}^{*}\right\|^{2} p_{43}^{i-1}\left(\mathbf{s}_{q}\right)-\left\|\sum_{q=1}^{Q} \mathbf{H}_{2} \mathbf{s}_{q}^{*} p_{43}^{i-1}\left(\mathbf{s}_{q}\right)\right\|^{2}+\sigma^{2},
$$

$$
\begin{aligned}
& u_{1,3}^{i}=\sum_{q=1}^{Q} \mathbf{H}_{1} \mathbf{s}_{q} p_{11}^{i-1}\left(\mathbf{s}_{q}\right)+\sum_{q=1}^{Q} \mathbf{H}_{2} \mathbf{s}_{q} p_{12}^{i-1}\left(\mathbf{s}_{q}\right), \\
& \sigma_{1,3}^{i}=\sum_{q=1}^{Q}\left\|\mathbf{H}_{1} \mathbf{s}_{q}\right\|^{2} p_{11}^{i-1}\left(\mathbf{s}_{q}\right)-\left\|\sum_{q=1}^{Q} \mathbf{H}_{1} \mathbf{s}_{q} p_{11}^{i-1}\left(\mathbf{s}_{q}\right)\right\|^{2} \\
& +\sum_{q=1}^{Q}\left\|\mathbf{H}_{2} \mathbf{s}_{q}\right\|^{2} p_{12}^{i-1}\left(\mathbf{s}_{q}\right)-\left\|\sum_{q=1}^{Q} \mathbf{H}_{2} \mathbf{s}_{q} p_{12}^{i-1}\left(\mathbf{s}_{q}\right)\right\|^{2}+\sigma^{2}, \\
& u_{2,3}^{i}=\sum_{q=1}^{Q} \mathbf{H}_{2} \mathbf{s}_{q}^{*} p_{21}^{i-1}\left(\mathbf{s}_{q}\right)-\sum_{q=1}^{Q} \mathbf{H}_{1} \mathbf{s}_{q}^{*} p_{22}^{i-1}\left(\mathbf{s}_{q}\right), \\
& \sigma_{2,3}^{i}=\sum_{q=1}^{Q}\left\|\mathbf{H}_{2} \mathbf{s}_{q}^{*}\right\|^{2} p_{21}^{i-1}\left(\mathbf{s}_{q}\right)-\left\|\sum_{q=1}^{Q} \mathbf{H}_{2} \mathbf{s}_{q}^{*} p_{21}^{i-1}\left(\mathbf{s}_{q}\right)\right\|^{2} \\
& +\sum_{q=1}^{Q}\left\|\mathbf{H}_{1} \mathbf{s}_{q}^{*}\right\|^{2} p_{22}^{i-1}\left(\mathbf{s}_{q}\right)-\left\|\sum_{q=1}^{Q} \mathbf{H}_{1} \mathbf{s}_{q}^{*} p_{22}^{i-1}\left(\mathbf{s}_{q}\right)\right\|^{2}+\sigma^{2}, \\
& u_{3,3}^{i}=-\sum_{q=1}^{Q} \mathbf{H}_{3} \mathbf{s}_{q}^{*} p_{31}^{i-1}\left(\mathbf{s}_{q}\right)+\sum_{q=1}^{Q} \mathbf{H}_{4} \mathbf{s}_{q} p_{32}^{i-1}\left(\mathbf{s}_{q}\right), \\
& \sigma_{3,3}^{i}=\sum_{q=1}^{Q}\left\|\mathbf{H}_{3} \mathbf{s}_{q}^{*}\right\|^{2} p_{31}^{i-1}\left(\mathbf{s}_{q}\right)-\left\|\sum_{q=1}^{Q} \mathbf{H}_{3} \mathbf{s}_{q}^{*} p_{31}^{i-1}\left(\mathbf{s}_{q}\right)\right\|^{2} \\
& +\sum_{q=1}^{Q}\left\|\mathbf{H}_{4} \mathbf{s}_{q}\right\|^{2} p_{32}^{i-1}\left(\mathbf{s}_{q}\right)-\left\|\sum_{q=1}^{Q} \mathbf{H}_{4} \mathbf{s}_{q} p_{32}^{i-1}\left(\mathbf{s}_{q}\right)\right\|^{2}+\sigma^{2}, \\
& \sigma_{4,3}^{i}=\sum_{q=1}^{Q}\left\|\mathbf{H}_{4} \mathbf{s}_{q}\right\|^{2} \mathbf{s}_{41}^{*}\left\|^{2} p_{42}^{i-1}\left(\mathbf{s}_{q}\right)-\right\| \sum_{q=1}^{Q} \mathbf{s}_{3} \mathbf{s}_{q}^{*} p_{42}^{i-1}\left(\mathbf{s}_{q}\right) \|_{q}+\sum_{q=1}^{Q}+\sigma^{2} . \\
& u_{4} \mathbf{H}_{4} \mathbf{s}_{q} p_{41}^{i-1}\left(\mathbf{s}_{q}\right)-\sum_{q=1}^{Q} \mathbf{H}_{3} \mathbf{s}_{q}^{*} p_{42}^{i-1}\left(\mathbf{s}_{q}\right), \\
& +-1 \\
& \left.+\mathbf{s}_{q}\right) \|^{2}
\end{aligned}
$$

\section{REFERENCES}

[1] H. Jafarkhani, "Space-time coding: Theory and practice," Cambridge, U.K.: Cambridge Univ. Press, 2005.

[2] S. Alamouti. "A simple transmit diversity technique for wireless communications," IEEE J. Sel. Areas Commun., vol. 16, no. 8, pp. 1451-1458, Oct. 1998.

[3] J. Li, D. Chen, D. Qu, Y. Zhang, and T. Jiang, "Receiver design for Alamouti coded FBMC system in highly frequency selective channels," IEEE Trans. Broadcasting, vol. 65, no. 3, pp. 601-608, Sep. 2019.

[4] T. Jiang, C. Ni, L. Guan, and Q. Qi, "Peak-to-average power ratio reduction in Alamouti multi-input-multi-output orthogonal frequency division multiplexing systems without side information using phase offset based-partial transmit sequence scheme," IET Communications, vol. 8, no. 5, pp. 564-570, Mar. 2014.

[5] V. Tarokh, N. Seshadri, and A. R. Calderbank, "Space-time codes for high data rate wireless communication: Performance criterion and code construction," IEEE Trans. Inf. Theory, vol. 44, no. 2, pp. 744-765, Mar. 1998.

[6] V. Tarokh, H. Jafarkhani, and A. R. Calderbank, "Space-time block codes from orthogonal designs," IEEE Trans. Inf. Theory, vol. 45, no. 5, pp. 1456-1467, July 1999.

[7] W. Su, X. Xia, and K. J. Liu, "A systematic design of high-rate complex orthogonal space-time block codes," IEEE Commun. Lett., vol. 8, no. 6, pp. 380-382, June 2004.

[8] H. Jafarkhani, "A quasi-orthogonal space-time block code," IEEE Trans. Commun., vol. 49, no. 1, pp. 1-4, Jan. 2001. 
[9] W. Su and X. Xia, "Signal constellations for quasi-orthognal space time block codes with full diversity," IEEE Trans. Inf. Theory, vol. 50, no. 10, pp. 2331-2347, Oct. 2004.

[10] L. A. Dalton and C. N. Georghiades, "A full-rate, full-diversity fourantenna quasi-orthogonal space-time block code," IEEE Trans. Wireless Commun., vol. 4, no. 2, pp. 363-366, Mar. 2005.

[11] W. Song, M. Lee, M. Matalgah and Y. Guo. "Quasi-orthogonal spacetime block codes designs based on jacket transform," Jounral of Communication and Networks, vol. 12, no. 3, pp. 240-245, June 2010.

[12] Z. Lei, C. Yuen and F. Chin, "Quasi-orthogonal space-time block codes for two transmit antennas and three time slots," IEEE Trans. Wireless Commun., vol. 10, no. 6, pp. 1983-1991, June 2011.

[13] L. Wang and Z. Chen. "Spatially modulated diagonal space time code," IEEE Commun. Lett., vol. 19, no. 7, pp. 1245-1248, July, 2015.

[14] V. Vakilian and H. Mehrpouyan, "High rate and low-complexity spacetime block codes for $2 \times 2$ MIMO systems," IEEE Commun. Lett., vol. 20, no. 6, 1227-1230, June 2016

[15] X. Li, R. Fan, X. Ma, J. An, and T. Jiang, "Secure space-time communications over rayleigh flat fading channels," IEEE Trans. Wireless Commun., vol. 15, no. 2, pp. 1491-1504, Feb. 2016.

[16] P. Liu, S. Jin, T. Jiang, Q. Zhang, and M. Matthaiou, "Pilot power allocation through user grouping in multi-cell massive MIMO systems," IEEE Trans. Commun., vol. 65, no. 4, pp. 1561-1574, Apr. 2017.

[17] T. Zhang, C. Wen, S. Jin, and T. Jiang, "Mixed-ADC massive MIMO detectors: Performance analysis and design optimization," IEEE Trans. Wireless Commun., vol. 15, no. 11, pp. 7738-7752, Nov. 2016.

[18] S. Li, J. Zhang and X. Mu, "Noncoherent massive space-time block codes for uplink network communication," IEEE Trans. Veh. Techno., vol. 67, no. 6, pp. 5013-5027, Mar. 2018.

[19] M. K. Arti, "OSTBC transmission in large MIMO systems," IEEE Commun. Lett., vol. 20, no. 11, pp. 2308-2311, Nov. 2016.

[20] X. Xia and X. Gao, "A space-time code design for omnidirectional transmission in massive MIMO Systems," IEEE Wireless Commun. Lett., vol. 5, no. 5, pp. 512-515, Aug. 2016.

[21] X. Meng, X. Xia and X. Gao, "Omnidirectional space-time block coding for common information broadcasting in massive MIMO systems," IEEE Trans. Wireless Commun., vol. 17, no. 3, Mar. 2018.

[22] C. Liu, X. Xia, Y. Li, X. Gao and H. Zhang, "Omnidirectional Quasiorthogonal space-time block coding massive MIMO systems," IEEE Commun. Lett., vol. 23, no. 9, pp. 1621-1625, Sep. 2019.

[23] S. Li, J. Zhang and X. Mu, "Double full diversity massive unitary spacetime codes for MIMO Channels," IEEE Trans. Veh. Technol., vol. 68, no. 4, pp. 3686-3701, Apr. 2019.

[24] A. Morsali, S. S. Hosseini, B. Champagne, and X. W. Chang, "Design criteria for omnidirectional STBC in massive MIMO systems," IEEE Wireless Commun. Lett., vol. 8, no. 5, pp. 1435-1439, Oct. 2019.

[25] R. Mesleh, H. Haas, S. Sinanovic, C. W. Ahn, and S. Yun, "Spatial modulation," IEEE Trans. Veh. Technol., vol. 57, no. 4, pp. 2228-2241, Jul. 2008.

[26] M. Di Renzo, H. Haas, and P. M. Grant, "Spatial modulation for multiple-antenna wireless systems: A survey," IEEE Commun. Mag., vol. 49, no. 12, pp. 182-191, Dec. 2011.

[27] M. Di Renzo, H. Haas, A. Ghrayeb, S. Sugiura, and L. Hanzo, "Spatial modulation for generalized MIMO: challenges, opportunities and implementation," Proceedings of the IEEE, vol. 102, no. 1, pp. 56103, Jan. 2014

[28] P. Yang, Y. Xiao, Y. L. Guan, K. V. S. Hari, A. Chockalingam, S Sugiura, H. Haas, M. Di Renzo, C. Masouros, Z. Liu, L. Xiao, S. Li, and L. Hanzo, "Single-carrier spatial modulation: A promising design for large-scale broadband antenna systems," IEEE Commun. Surveys Tuts., vol. 18, no. 3, pp. 1687-1716, Feb. 2016.

[29] L. Xiao, P. Xiao, Y. Xiao, H. Hass, A. Mohamed, and L. Hanzo, "Compressive sensing assisted generalized quadrature spatial modulation for massive MIMO," IEEE Trans. Commun. vol. 67, no. 7, pp. 47954810, May 2019.
[30] E. Basar, "Index modulation techniques for $5 \mathrm{G}$ wireless networks," IEEE Commun. Mag., vol. 54, no. 7, pp. 168-175, July 2016.

[31] E. Basar, U. Aygolu, E. Panayirci and H. V. Poor, "Space-time block coded spatial modulation," IEEE Trans. Commun., vol. 59, no. 3, pp. 823-832, Mar. 2011.

[32] X. Li and L. Wang, "High rate space-time block coded spatial modulation with cyclic structure," IEEE Commun. Lett., vol. 18, no. 4, pp. 532-535, Apr. 2014

[33] C. Jeon and J. Lee, "Multi-strata space-time coded spatial modulation," IEEE Commun. Lett., vol. 19, no. 11, pp. 1945-1948, Nov. 2015.

[34] Y. Hua, G. Zhao, W. Zhao, and M. Jin, "Modified codewords design for space-time block coded spatial modulation," IET Commun., vol. 11, no. 2, pp. 249-257, Jan. 2017.

[35] M. C. Park, B. G. Jo and D. S. Han, "Double space-time transmit diversity with spatial modulation," IEEE Electronic Lett., vol. 51, no. 25, pp. 2155-2156, Dec. 2015.

[36] L. Xiao, Y. Xiao, L. You, P. Yang, S. Li and L. Hanzo, "Single-RF and Twin-RF spatial modulation for an arbitrary number of transmit antennas," IEEE Trans. Veh. Technol., vol. 67, no. 7, pp. 6311-6324, July 2018.

[37] L. Xiao, Y. Xiao, P. Yang, J. Liu, S. Li, and W. Xiang, "Space-time block coded differential spatial modulation," IEEE Trans. Veh. Technol., vol. 66, no. 10, pp. 8821-8834, Oct. 2017.

[38] C. Wu, Y. Xiao, L. Xiao, P. Yang, and X. Lei, "Space-time block coded rectangular differential spatial modulation: System design and performance analysis", IEEE Trans. Commun., vol. 67, no. 9, pp. 65866597, May 2019.

[39] C. Xu, R. Rajashekar, N. Ishikawa, S. Sugiura and L. Hanzo, "SingleRF index shift keying aided differential space-time block coding," IEEE Trans. Signal Process. vol. 66, no. 3, pp. 773-788, Feb. 2018.

[40] C. Xu, P. Zhang, R. Rajashekar, N. Ishikawa, S. Sugiura, L. Wang and L. Hanzo, "Finite-cardinality single-RF differential space-time modulation for improving the diversity-throughput tradeoff," IEEE Trans. Commun., vol. 67 , no. 1 , pp. 318-335, Jan. 2019.

[41] N. Ishikawa, R. Rajashekar, C. Xu, S. Sugiura, and L. Hanzo, "Differential space-time coding dispensing with channel estimation approaches the performance of its coherent counterpart in the open-loop massive MIMO-OFDM downlink," IEEE Trans. Commun., vol. 66, no. 12, pp. 6190-6204, Dec. 2018.

[42] M. Le, V. Ngo, H. Mai, X. Nam, and M. Di. Renzo, "Spatially modulated orthogonal space-time block codes with non-vanishing determinants," IEEE Trans. Commun., vol. 62, no. 1, pp. 85-99, Jan. 2014.

[43] L. Xiao, P. Xiao, C. Xu, I. Hemadeh, D. Mi and W. Hao, "Generalized space-time block coded spatial modulation systems," IEEE PIMRC, Istanbul, Turkey, 2019.

[44] L. Xiao, P. Yang, Y. Xiao, S. Fan, M. Renzo, and S. Q. Li, "Efficient compressive sensing detectors for generalized spatial modulation systems," IEEE Trans. Veh. Technol., vol. 66, no. 2, pp. 1284-1298, Feb. 2017.

[45] D. L. Donoho, A. Maleki, and A. Montanari, "Message passing algorithms for compressed sensing," Proc. Nat. Acad. Sci., vol. 106, pp. 18914-18919, 2009

[46] E. Basar, U. Aygolu, E. Panayici and H. V. poor, "Orthogonal frequency division multiplexing with index modulation," IEEE Trans. Signal Process., vol. 61. no. 22, Nov. 2013.

[47] L. Xiao, Y. Xiao, C. Xu, X. Lei, P. Yang, S. Li, and L. Hanzo, "Compressed-sensing assisted spatial multiplexing aided spatial modulation," IEEE Trans. Wireless Commun., vol. 17, no. 2, pp. 794-807, Feb. 2018.

[48] J. Hoydis, S. Brink, and M. Debbah, "Massive MIMO in the UL/DL of cellular networks: How many antennas do we need?" IEEE J. Sel. Areas Commun., vol. 31, no. 2, pp. 160-171, Jan. 2013. 